\title{
Intrinsic spin Hall conductivity in three-dimensional topological insulator/normal insulator heterostructures
}

\author{
V. N. Men'shov, ${ }^{1,2,3,4}$ I. A. Shvets, ${ }^{3,4}$ V. V. Tugushev, ${ }^{1,2,3}$ and E. V. Chulkov ${ }^{1,4,5}$ \\ ${ }^{1}$ Donostia International Physics Center (DIPC), P. de Manuel Lardizabal 4, 20018 San Sebastián, Basque Country, Spain \\ ${ }^{2}$ NRC Kurchatov Institute, Kurchatov Square 1, 123182 Moscow, Russia \\ ${ }^{3}$ Tomsk State University, 634050 Tomsk, Russia \\ ${ }^{4}$ Saint Petersburg State University, 198504 Saint Petersburg, Russia \\ ${ }_{5}^{5}$ Departamento de Física de Materiales UPV/EHU, CFM-MPC UPV/EHU, 20080 San Sebastián/Donostia, Basque Country, Spain
}

(Received 9 April 2017; revised manuscript received 9 June 2017; published 1 August 2017)

\begin{abstract}
Here we study how interface and edge perturbations as well as a size effect can be used to manipulate the transport properties in semiconductor heterostructures where a thin film of three-dimensional topological insulator (TI) is sandwiched by normal insulator (NI) slabs. Within the framework of the NI/TI/NI trilayer model based on a continual scheme, we argue that characteristics of electron states in the TI film (energy spectrum, envelope function profile, the Berry curvature, etc.) are controlled by the film thickness and TI/NI interface potential whose variation can lead to the modification of topological properties of the system. Calculating a spin Hall response for the NI/TI/NI trilayer infinite in the interface plane, we find that a series of quantum transitions between topological insulating phase and trivial band insulator phase can be induced by tuning both the film thickness and the interface potential. We draw in detail the corresponding phase diagram of the NI/TI/NI trilayer, which is controlled by change of the sign of either the hybridization gap or the dispersion parameter. To quantify the edge effect, we formulate a model of the half-infinite in the interface plane NI/TI/NI trilayer, which describes evanescent edge states and provides the necessary conditions under which they exist. It is found that the presence of the in-gap edge states is ambiguously determined by the phase of the TI film. Our findings provide a useful guide in choosing the relevant material parameters to facilitate the observation of quantum spin Hall effect in the TI/NI heterostructures.
\end{abstract}

DOI: 10.1103/PhysRevB.96.075302

\section{INTRODUCTION}

From the viewpoint of the application perspective of solid-state materials with unusual electron properties for spintronic devices, design and fabrication of semiconductor heterostructures comprising three-dimensional (3D) topological insulator (TI) thin films interfaced with normal insulator (NI) films is undoubtedly a promising way [1-8]. The specific character of the bulk electron structure of 3D TI gives rise to electron states appearing at the TI/NI interfaces, where the topological invariant changes. These states are often said to be "topologically protected." They are notable for their massless Dirac-like spectrum and spin-momentum locking [9-11] and therefore deemed to play an important role in physics of the $\mathrm{TI} / \mathrm{NI}$ heterostructures. In contrast to a free TI surface exposed to air, the interfacial states buried inside the heterostructure are protected from surrounding environment. Albeit, the choice of an appropriate NI material, which does not destroy the specifics of topological states, is critical. Indeed, the contact between TI and NI can produce intermixing of wave-functions of these materials at the interface, charge transfer and crystal lattice distortion or may induce various proximity effects. These factors, which depend on the combination of the TI and NI materials and heterostructure engineering, influence the characteristics of the electron states at the interface. Moreover, when the 3D TI film is sufficiently thin, an electron state with a gapped energy spectrum occurs due to the hybridization between the interface states originated from opposite boundaries of the TI film. The characteristics of such a state depend strongly on both the finite-size film and the interface potential. Thus, the heterostructure design may be used as an efficient tool to manipulate the electron properties of the TI film, in particular, topology and dimensionality. This creates opportunities to drive electron transport phenomena such as quantum spin Hall effect (QSHE) in the TI/NI heterostructures devices [10,12-14]. Note that electron transport in the QSHE regime with insulating bulk indeed manifests itself due to conducting helical edge states which propagate along onedimensional (1D) channels occurring at edges of the TI thin film $[9,10,15]$. These gapless (metallic) edge states that lie inside the hybridization gap provide a quantized conductance in the ballistic limit. However, engineering of the spintronic devices based on the TI/NI heterostructures faces a challenge to achieve their desirable functionality. One of the major obstacles to use the TI/NI heterostructures is a lack of clear understanding of the role of the interfacial and edge boundaries in transport phenomena.

A large number of spectroscopy, transport and magnetotransport studies on the 3D TI thin films grown by molecular beam epitaxy on the NI substrates have reported on a variety of properties depending on the type of the substrate used and the film thickness [16-23]. In particular, these studies provide evidence for evolution of the electron states under the topological phase transitions emerging in the TI films. Similar observations have been made for the superlattices consisting of alternating layers of the 3D TI material, such as $\mathrm{Bi}_{2} \mathrm{Se}_{3}$ or $\mathrm{Sb}_{2} \mathrm{Te}_{3}$, and the NI material, such as $\mathrm{PbSe}$ or GeTe [3,4,24]. Just recently, Belopolski et al. [25] have synthesized a multilayer heterostructure stacking together thin layers of the TI $\mathrm{Bi}_{2} \mathrm{Se}_{3}$ and the NI $\mathrm{In}_{x} \mathrm{Bi}_{2-x} \mathrm{Se}_{3}$ and demonstrated that the heterostructure can be driven through a topological phase transition by changing its composition. 
There exist several theoretical research on the finite-size effect in the thin TI films [14,26-34]. The transitions between the QSHE insulator and trivial band insulator phases have been explored as being driven by alternation of the hybridization gap caused by the 3D TI film thickness [14,30-34]. At the same time, a proper description of the boundary effects in the TI/NI heterostructures is almost absent, with few exceptions [35-40]. The development of the quantum transport theory in these systems requires to answer some principal questions. What is the combined effect of the TI/NI interfaces and the finite thickness of the TI film on electron properties of the system? Can an emergent QSHE regime be specifically induced by the TI/NI interfaces? To what extent can the heterostructure edges control the existence and characteristics of the electron edge states? One needs thorough understanding and proper description of finite-size and boundary effects to predict the quantum conductivity behavior in the TI/NI heterostructures.

In the present work, to consider the principal aspects of the problem, we propose the model of the NI/TI/NI trilayer that can be reckoned as a fundamental building block of the TI/NI heterostructure. Within the framework of continual approach, the influence of the NI slabs on electron states in the sandwiched 3D TI spacer film is taken into consideration through the boundary conditions specified by the effective interface potential (IP), which respects time-reversal symmetry. Such analytic scheme has been developed and successfully used in the previous investigations [37-40] to show how the changes in the IP could modify the in-gap bound states at the TI/NI interface. In the present theoretical work, we unveil that there are two distinct microscopic mechanisms to drive the transition between the trivial insulator phase and the Hall insulator phase in the NI/TI/NI trilayer. One mechanism emphasizes, following Refs. [14,30-32], finite thickness of the TI film. While another mechanism stresses that just IP can induce such topological transition in the trilayer. Here we reconcile both mechanisms by constructing, for the first time, a topological phase diagram of the system. To verify whether the NI/TI/NI trilayer exhibits the quantized spin Hall conductance we specially focus on an analysis of the Dirac-like states localized at the edges of the sample bounded in the interface plane. We reveal that the quantized bulk response and the edge states, which exemplify spin Hall conductivity in the TI/NI heterostructures, are quite sensitive to the confinement factors such as the film thickness and the conditions at its boundaries. These facts clearly indicate the limits of the feasibility of the QSHE regime in real samples. At the same time, our results outline pathways of designing the heterostructures with given spin-transport properties by using available TI and NI materials.

The rest of the paper is organized as follows. In Sec. II, to study the NI/TI heterostructures, we formulate an approach based on the formalism of effective interface and edge potentials within the continuum scheme. In Sec. III, introducing a proper boundary problem at the interfaces, we explore the model of the NI/TI/NI trilayer which is infinite along the interface plane. We analyze electron energy levels and envelope function profiles in dependence on the TI film thickness and the IP strength. In Sec. IV we derive the effective 2D Hamiltonian for the NI/TI/NI trilayer and thoroughly describe, both analytically and numerically, the dependence of the Hamiltonian parameters as well as the energy spectrum on the IP strength, the film thickness and the TI material characteristics. In Sec. V, we demonstrate that a series of quantum phase transitions between the trivial band insulator and the spin Hall insulator can be driven by tuning either the thickness or the IP strength. At this point, we draw the corresponding phase diagram of the NI/TI/NI trilayer in detail. In Sec. VI, we systematically explore the edge states for the NI/TI/NI trilayer bounded in the interface plane to check their existence in topologically distinct phases under given value of an effective edge potential at the edge. In Sec. VII we discuss our theoretical findings and comment on a possibility to observe QSHE. In Sec. VIII, we summarize the main results of the work.

\section{MODEL OF HETEROSTRUCTURE COMPOSED OF TOPOLOGICAL AND NORMAL INSULATORS}

We consider the layered heterostructure in which a thin film of $3 \mathrm{D} \mathrm{TI}$ is in contact with NI. The typical example of such heterostructure is an NI/TI/NI trilayer consisting of a 3D TI film (for instance, $\mathrm{Bi}_{2} \mathrm{Se}_{3}$ single crystal film of a few quintuple-layer thickness) lying on an NI substrate and covered with an NI overlayer. Such trilayer can be regarded as a building block of the TI/NI heterostructure.

The TI and NI films are separated one from the other by interior boundaries (TI/NI interfaces) and also have external boundaries with surrounding media (edges of NI and TI films). In principle, electron structure of such a system is very complex and contains both itinerant and localized electron states of different types. Strictly speaking, to fully capture this electron structure it is necessary to use numerical methods. However, in certain situations it is possible to reduce the problem to a qualitative analysis of electron structure of some set of electron states presenting a particular interest in the framework of the model Hamiltonian. For example, if there exists a set of electron states inside the TI spacer, which are well separated in energy from the states inside the NI slabs, one can imitate the effect of TI/NI interfaces on the electron states inside TI introducing an external static IP.

The energy of the relevant electron states in the $3 \mathrm{D}$ TI film, which is confined along all three directions, generally reads

$$
\Omega=\int d \mathbf{r} \Theta^{\dagger}(\mathbf{r})[\mathbb{H}(-i \nabla)+\mathbb{W}(\mathbf{r})] \Theta(\mathbf{r}),
$$

where $\mathbb{H}(-i \nabla)$ is the Hamiltonian of the 3D TI bulk materials, and $\Theta(\mathbf{r})$ is an envelope function (EF). The influence of the surrounding media through the TI/NI interfaces and edges is treated by means of the confining potential $\mathbb{W}(\mathbf{r})$.

The paradigmatic scheme proposed to describe the long wavelength bulk states of the narrow-gap semiconductor materials of $\mathrm{Bi}_{2} \mathrm{Se}_{3}$-type is based on the band basis, $u_{\Gamma}=$ $\{|+\uparrow\rangle,|-\uparrow\rangle,|+\downarrow\rangle,|-\downarrow\rangle\}$, formed by four hybridized states of the Se and $\mathrm{Bi} p_{z}$-orbitals [41,42]. Within the kp scheme, the model Hamiltonian of 3D TI is a power expansion with respect to the wave vector $\mathbf{k}$ around the $\Gamma$ point $(\mathbf{k}=0)$, whose coefficients are constrained by parity and time-reversal symmetries. In what follows, for the sake of calculating simplicity, but without a loss of generality, we use the particle-hole symmetric and isotropic $4 \times 4$ Hamiltonian in 
the form

$$
\mathbb{H}(\mathbf{k})=\Xi(\mathbf{k}) \tau_{z} \otimes \sigma_{0}+A \tau_{x} \otimes(\boldsymbol{\sigma} \cdot \mathbf{k}),
$$

where $\Xi(\mathbf{k})=\Xi_{0}-B k^{2}, 2 \Xi_{0}$ is a band gap, $B$ describes a band curvature, and $\sigma_{\alpha}$ and $\tau_{\alpha}(\alpha=0, x, y, z)$ denote the Pauli matrices in the spin and orbital space, respectively. The Hamiltonian Eq. (2) captures the remarkable feature of the band structure: the inverted order in energy of the basis states near $\mathbf{k}=0$ due to strong spin-orbit coupling, which arises under the condition $\Xi_{0} \mathrm{~B}>0$, correctly characterizes the topological nature of the system. The operator $\mathbb{H}(\mathbf{k} \rightarrow-i \nabla)$, determined in Eq. (2) acts in the space of EFs of Bloch waves that are represented by spinors $\Theta(\mathbf{r})=\left(\theta_{1}(\mathbf{r}), \theta_{2}(\mathbf{r}), \theta_{3}(\mathbf{r}), \theta_{4}(\mathbf{r})\right)^{\text {tr }}$ in the basis $u_{\Gamma}$, the superscript tr denotes the transpose operation. The EF components $\theta_{j}(\mathbf{r})$ are presumed to be smooth functions inside the TI film.

In this approach, we do not consider the behavior of the electron wave function out of the TI film. However, in practice, there are some requirements for the NI constituent of the heterostructure, which must be fulfilled to preserve the topologically relevant states in the TI film. So, the NI materials (for instance, substrates and overlayers in the trilayer) should have a large bulk band gap as compared with that of 3D TI material, $2 \Xi_{0}$. Furthermore, the topologically relevant electron states featured by Eq. (2) should be well separated in energy from the bulk states of NI as well as from trivial localized states (for example, the Schokley-type states) often appearing at semiconductor interfaces. Also, the chemical potential $\mu$ of the system is assumed to be inside the TI bulk band gap, $|\mu|<\Xi_{0}$.

On the one hand, the bottom and top surfaces of the TI film in the NI/TI/NI trilayer are interfaced with the NI material. We suppose that the TI film occupies the space $|z|<l$ along the heterostructure growth direction $\mathbf{z}$ and the interfaces are perfectly flat. On the other hand, the TI film is bounded in the interface plane $(x, y)$ by the edges (for example, in the case of a sample designed in a Hall bar geometry) meeting vacuum or some topologically trivial medium at a circumference $\Upsilon(x, y)=0$. Then the confining potential in Eq. (1), which experienced by electron inside the TI film, can be represented as $\mathbb{W}(\mathbf{r}) \approx d \mathbb{U} \delta(z+l)+d \mathbb{U} \delta(z-l)+t \mathbb{V} \delta[\Upsilon(x, y)]$, where $\delta(z)$ is the $\delta$ function. Here we adopt the local approximation, as long as $\mathrm{EF} \Theta(\mathbf{r})$ is slowly varying over the scale $\sim d, t$ near the boundaries where potential $\mathbb{W}(\mathbf{r})$ is localized ( $d$ and $t$ are of the order of a few lattice spacings). Following the concept of Refs. [37-39], we have introduced IP $\mathbb{U} \delta(z \pm l)$, which reflects such effects as, for instance, charge density transfer and/or intermixing of atomic orbitals at the TI/NI interface. The edges of the TI film can be terminated with dangling bonds which may be oxidized during the fabrication of the heterostructure. In the present continual approach, we simulate the influence of the edges on electron states in the TI film by means of the term $\mathbb{V} \delta[\Upsilon(x, y)]$, which is hereinafter referred to as an edge potential (EP). In the basis $u_{\Gamma}$, IP and EP are the $4 \times 4$ matrices. Authors of Ref. [43] using universal physical requirements derived such matrix in the most general form. As microscopic details of a real interface are unknown, in following calculations we approximate IP and EP by diagonal matrices.

\section{MODEL OF THE UNBOUNDED NI/TI/NI TRILAYER}

At the beginning we neglect the edge effects and consider the trilayer being infinite in the interface plane $(x, y)$. For simplicity, the TI film is assumed to be bordered both above $(z=l)$ and below $(z=-l)$ by the same NI material; i.e., the NI/TI/NI trilayer is symmetric. IP is regarded as being spin-independent so it can be parameterized only by two real constants $U_{1}$ and $U_{2}$ through the diagonal matrix $\mathbb{U}=$ $\operatorname{diag}\left\{U_{1}, U_{2}, U_{1}, U_{2}\right\}$.

We make use of a variational procedure for the relevant energy functional:

$$
\begin{aligned}
\mathbb{F}\left\{\Theta^{\dagger}, \Theta\right\}= & \int_{-l}^{l} d z \Theta^{\dagger}(\boldsymbol{\kappa}, z)\left[\mathbb{H}\left(\boldsymbol{\kappa},-i \partial_{z}\right)\right. \\
& +d \mathbb{U}(\boldsymbol{\kappa}) \delta(|z|-l)-\mathbb{I} E] \Theta(\boldsymbol{\kappa}, z),
\end{aligned}
$$

where the energy $E$ plays a role of the Lagrange multiplier, II is an unit $4 \times 4$ matrix, and $\partial_{z}=\partial / \partial z$. The functional Eq. (3) is determined in the class of the smooth and continuous EFs inside the 3D TI film and includes the effective IP. Since the system remains translational symmetry in the $(x, y)$ plane, the in-plane wave-vector $\kappa=\left(k_{x}, k_{y}\right)$ is a good quantum number. Therefore, we can determine the functional for each EF $\boldsymbol{\kappa}$ mode, $\Theta(\kappa, z)$. Varying $\mathbb{F}\left\{\Theta^{\dagger}, \Theta\right\}$ with respect to the function $\Theta^{\dagger}$ yields the Euler equations inside the film, $|z|<l$, and the boundary conditions imposed on EF at the interface at $|z|= \pm l$. The corresponding equations in the compact form are

$$
\left[\mathbb{H}\left(\kappa,-i \partial_{z}\right)-\mathbb{I} E\right] \Theta(\kappa, z)=0,
$$

$$
\left.i \frac{\delta \mathbb{H}\left(\kappa,-i \partial_{z}\right)}{\delta\left(-i \partial_{z}\right)} \Theta(\kappa, z)\right|_{|z|= \pm l}=\mp 2 d \mathbb{U}(\kappa) \Theta(\kappa, \pm l) .
$$

In our approach, the boundary conditions Eq. (5) involve relations between the EFs of Bloch waves on both sides of the TI/NI interface via the effective IP [37]. The solution of the boundary problem of Eqs. (4) and (5) answers the important question on how the IP located just at the boundaries affects the electron states inside the 3D TI film. Here we go beyond the open boundary condition, where the state has zero amplitude at the interfaces, $\Theta( \pm l)=0$. So this condition, on the one hand, does not look like a physically natural one and, on the other hand, reduces significantly the capability of a model approach.

For the TI thin film confined in $\mathbf{z}$ direction, the electron motion along this direction is quantized to discrete levels related to quantum well-like $2 \mathrm{D}$ subbands in the spectrum, $E(\kappa)$. In the case of the Hamiltonian Eq. (2), a search for the exact eigen functions $\Theta(\kappa, z)$ and energies $E(\boldsymbol{\kappa})$ is a formidable problem. The task Eqs. (4) and (5) can be solved analytically at $\boldsymbol{\kappa}=0$ when the Hamiltonian acquires a block-diagonal form: $\mathbb{H}\left(0,-i \partial_{z}\right)=\operatorname{diag}\left\{\mathcal{H}^{+}\left(-i \partial_{z}\right), \mathcal{H}^{-}\left(-i \partial_{z}\right)\right\}$ with

$$
\mathcal{H}^{\sigma}\left(-i \partial_{z}\right)=\tau_{z}\left(B \partial_{z}^{2}+\Xi_{0}\right)+i \tau_{x} \sigma A \partial_{z},
$$

where the superscript $\sigma=+/-$ means an up/down projection of electron spin onto the quantization axis. The lower block $\mathcal{H}^{-}$is the time reversal of the upper block $\mathcal{H}^{+}$. The bispinorfunctions $\varphi^{\sigma}(z)$ and $\chi^{\sigma}(z)$, presenting the components of $\mathrm{EF}$ 
$\Theta(\kappa=0, z)$, obey the boundary problem:

$$
\begin{gathered}
\mathcal{H}^{\sigma}\left(-i \partial_{z}\right) \varphi^{\sigma}(z)=E_{\varphi} \mathcal{I} \varphi^{\sigma}(z), \\
\mathcal{H}^{\sigma}\left(-i \partial_{z}\right) \chi^{\sigma}(z)=E_{\chi} \mathcal{I} \chi^{\sigma}(z), \\
\left.\mathcal{G}^{\sigma}\left(-i \partial_{z}, z\right) \varphi^{\sigma}(z)\right|_{z= \pm l}=0, \\
\left.\mathcal{G}^{\sigma}\left(-i \partial_{z}, z\right) \chi^{\sigma}(z)\right|_{z= \pm l}=0,
\end{gathered}
$$

where the operator $\mathcal{G}^{\sigma}$ is given by

$$
\begin{aligned}
\mathcal{G}^{\sigma}\left(-i \partial_{z}, z\right)= & \left\{-\tau_{0} \operatorname{sgn}(z) d\left(U_{1}+U_{2}\right)+\tau_{z}\left[2 B \partial_{z}\right.\right. \\
& \left.\left.-\operatorname{sgn}(z) d\left(U_{1}-U_{2}\right)\right]+i \tau_{x} \sigma A\right\} .
\end{aligned}
$$

Here $\mathcal{I}$ is an unit $2 \times 2$ matrix.

Due to the space symmetry of the system along the $\mathbf{z}$ axis, the trial solutions of the task Eqs. (7) and (8) can be represented as superpositions of (the even functions) $\cosh \left(q_{\varphi 1,2} z\right)$ and $\cosh \left(q_{\chi 1,2} z\right)$ for bound states or (the odd functions) $\sinh \left(q_{\varphi 1,2} z\right)$ and $\sinh \left(q_{\chi 1,2} z\right)$ for antibound states, where the corresponding superposition coefficients are functions of $l$ and $U_{1,2}$. The momenta $q_{\varphi 1,2}=q_{1,2}\left(E_{\varphi}\right)$ and $q_{\chi 1,2}=q_{1,2}\left(E_{\chi}\right)$, specifying a scale of the EF space variation, are connected with the energy via the relation

$$
\left[q_{1,2}(E)\right]^{2}=-k_{m}^{2} \pm \frac{\sqrt{E^{2}-E_{m}^{2}}}{B} .
$$

Here the following notations are used: $k_{m}^{2}=(1-2 \lambda) \Xi_{0} / B$ and $E_{m}^{2}=4 \lambda(1-\lambda) \Xi_{0}^{2}$, as well as $\lambda=A^{2} / 4 B \Xi_{0}$ for the parameter featuring the bulk band structure of 3D TI. In the case of $\lambda<1$, the bulk spectrum of the Hamiltonian Eq. (2) takes a "camelback" shape with minimal gap of $2 E_{m}$ at nontrivial momentum $k_{m}$. As for the momenta $q_{1,2}(E)$, the situation is tricky at $\lambda<1$. When $E^{2}>\Xi_{0}^{2}$, one has $q_{1}=\operatorname{Re}$ and $q_{2}=\mathrm{Im}$. When $\Xi_{0}^{2}>E^{2}>E_{m}^{2}$ and $1>\lambda>1 / 2$, both the momenta, $q_{1}$ and $q_{2}$, are real. Under condition that $\Xi_{0}^{2}>E^{2}>E_{m}^{2}$ and $\lambda<1 / 2$, both the momenta, $q_{1}$ and $q_{2}$, are imaginary. If $E^{2}<E_{m}^{2}$, then both the momenta are complex and $q_{1}(E)=q_{2}^{*}(E)$.

The $\varphi$-state with the energy $E_{\varphi n}$ and the $\operatorname{EF} \varphi_{n}^{\sigma}(z)$ and the $\chi$-state with the energy $E_{\chi n}$ and the EF $\chi_{n}^{\sigma}(z)$ have opposite space parities, the index $n(n=0, \pm 1, \pm 2, \ldots)$ enumerates the discrete levels of the electron motion along $z$ axis in the film geometry (the well-known quantum size effect). Each of these levels is twofold degenerate in spin due to time-reversal symmetry. Clearly, the components of the $\varphi$ and $\chi$ states can be treated as the bonding and antibonding couplings of the states ascribed to top and bottom boundaries of the thick film. Inserting the trial solutions into Eqs. (7) and (8), it is straightforward to determine the superposition coefficients as well as the energies $E_{\varphi n}$ and $E_{\chi n}$. All these values directly depend on the IP matrix elements and the film thickness.

One can in principle obtain a complete set of the eigen discrete values for Eqs. (7) and (8). Figure 1 summarizes the behavior of the energy levels $E_{\varphi n}$ and $E_{\chi n}$ with increasing the TI film thickness at the different (fixed) IP strengths for two distinct cases $U_{1}=U_{2}$ [Fig. 1(a)] and $U_{1}=-U_{2}$ [Fig. 1(b)] at the relatively small $\lambda$. We use the dimensionless parameters $\tilde{l}=l \sqrt{\frac{\Xi_{0}}{B}}, \tilde{U}=\frac{d U}{\sqrt{B \Xi_{0}}}$. It is easy to separate the states into two groups; for the sake of convenience let us call them low-energy states and high-energy ones. The low-energy states, indexed by $n=0$, arise due to a nontrivial wave function topology of the bulk material. They are caused by an overlap of the Dirac-like states stemmed from the opposite interfaces of the TI film. Note that, depending on the film thickness and the IP strength, the energies $E_{\varphi 0}$ and $E_{\chi 0}$ may either remain inside the projected bulk band gap of $3 \mathrm{D} \mathrm{TI}, E_{\varphi, \chi 0}^{2}<E_{m}^{2}$, or merge in the projected bulk bands, $E_{\varphi, \chi 0}^{2}>E_{m}^{2}$. In any case, at fixed thickness, one has $\left|E_{\varphi, \chi 0}\right|<\left|E_{\varphi, \chi n}\right|$, where the index $|n| \geqslant 1$ relates to the high-energy states, which exist only either above the bottom of the projected bulk conduction band $(n \geqslant 1)$ or below the top of the projected bulk valence band $(n \leqslant-1)$, i.e., at $E_{\varphi, \chi n}^{2}>E_{m}^{2}$. The high-energy states are similar to the quantum well states of a trivial semiconductor film. The energy values $\left|E_{\varphi, \chi n}(l)\right|$ increase with decreasing $l$ as a result of the quantum well effect and can exceed $\Xi_{0}$ at $\operatorname{Re}\left(q_{1,2}\right) l<1$. We note also that the energy levels $E_{\varphi 0}$ and $E_{\chi 0}$ can merge into projected bulk bands, $E_{\varphi, \chi 0}^{2}>E_{m}^{2}$, i.e., the in-gap states are absent, under the condition $\operatorname{Re}\left(q_{1,2}\right) l \gtrsim 1$ in certain parameter region $\left(U_{1}, U_{2}\right)$, for example, such situation has place at $U_{1}=$ $U_{2}=U$ when $\widetilde{U} \approx 1$. The specific feature of the Hamiltonian Eq. (2) at $0<\lambda<1$ is the oscillating behavior of the eigenvalues of the $\varphi$ and $\chi$ low-energy states with the varying thickness, which results in the corresponding closing and reopening of the hybridization gap $2 \Delta_{0}(l)=E_{\varphi 0}(l)-E_{\chi 0}(l)$. Furthermore, as seen in Fig. 1, the energy levels of the high-energy states depend nonmonotonously on the thickness as well, which leads to that the states with opposite parity cross each other out of the projected bulk gap. These features in the spectrum of the NI/TI/NI trilayer (at $0<\lambda<1$ ), connected with a nontrivial topology of the Hamiltonian Eq. (2) and manifested in the complicated dependence of the levels $E_{\varphi n}$ and $E_{\chi n}$ on both the thickness and the IP strength, differ principally from those of the quantum well based on a trivial semiconductor. When the thickness of the NI material film exceeds greatly a lattice constant, nearly complete convergence of all quantum well subbands to bulk band continuum is achieved. In the case of the TI film, energy divergence between the quantum well-like states and the in-gap topological state increases with the thickness.

We are interested in study of the space profile of the low-energy states lying inside the projected bulk energy gap, which are of primary importance. Figure 2 displays a set of the EF module profiles of the $\varphi$ and $\chi$ states in the direction perpendicular to the boundaries for IP with $U_{1}=-U_{2}=U$ ranging from 0 to $\infty$ (the "hard-wall" boundary condition) at the fixed film thickness. The space distributions of even components and odd components of the bispinors $\varphi_{0}^{\sigma}(z)$ and $\chi_{0}^{\sigma}(z)$ coincide in pairs because such IP (with $U_{1}=-U_{2}$ ) does not break particle-hole symmetry of the Hamiltonian Eq. (2). In turn, at $U_{1} \neq-U_{2}$, each of the four components describing the low-energy state is specific in the profile. For example, Fig. 3 demonstrates an evolution of the profiles with the film thickness at fixed value of the IP matrix elements as $U_{1}=U_{2}=U$. When the thickness is large enough, the low-energy states are mostly peaked close to the interfaces and show an exponential decay over the scale $\sim\left[\operatorname{Re}\left(q_{1,2}\right)\right]^{-1}$ away from the interfaces accompanied by an oscillation with the period $\sim\left[\operatorname{Im}\left(q_{1,2}\right)\right]^{-1}$. Note that if IP is either strong, 

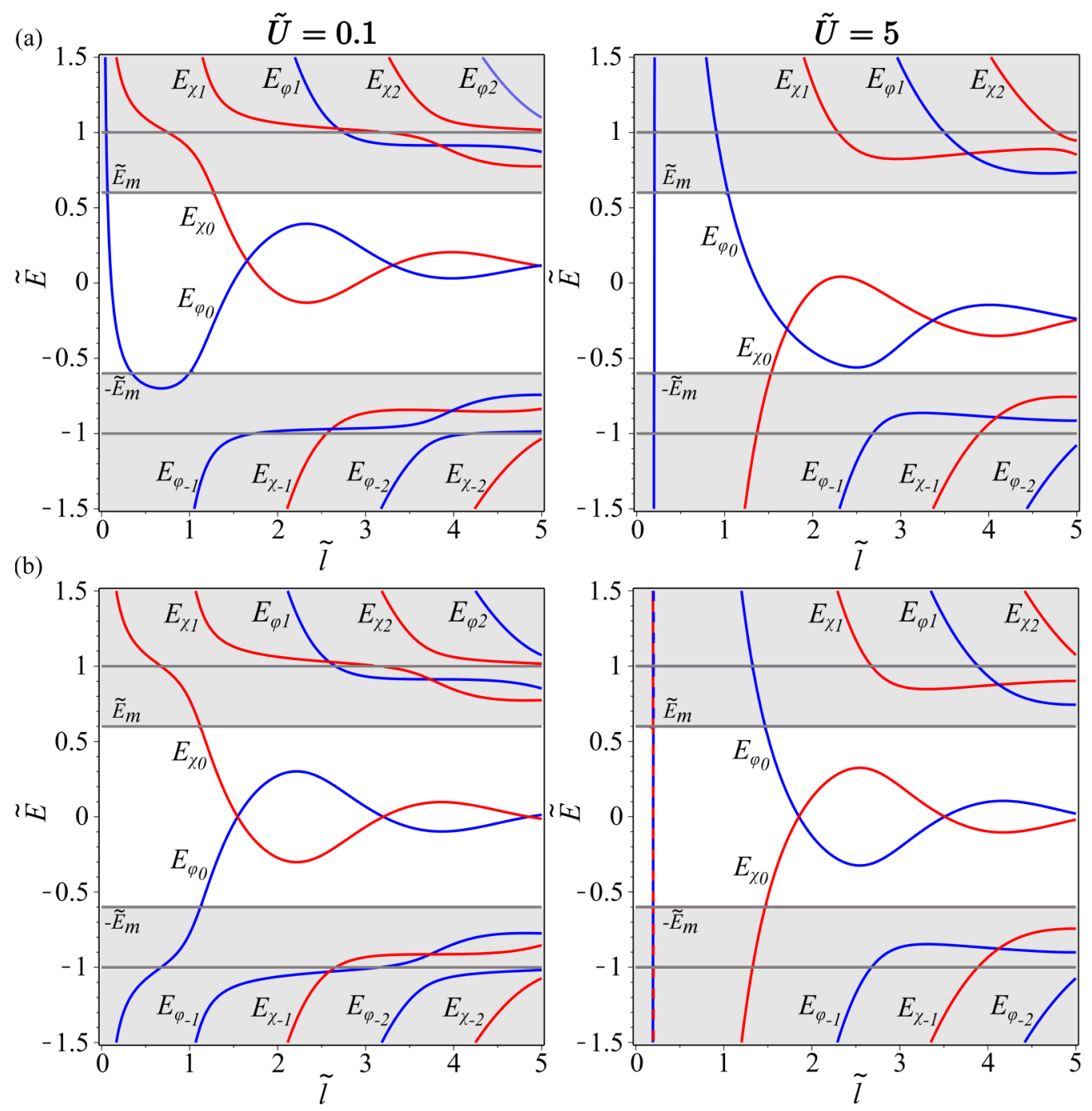

FIG. 1. Eigenvalues $E_{\varphi n}$ (blue lines) and $E_{\chi n}$ (red lines) of the $\varphi$ - and $\chi$-states calculated for a TI film as a function of the thickness $\tilde{l}$ with the fixed TI strength $\tilde{U}$. The definitions of relevant energies discussed in the text are indicated by the indices $n=0, \pm 1, \pm 2, \ldots$. Top panels show the result for the case $U_{1}=U_{2}=U$; whereas bottom panels show the result for $U_{1}=-U_{2}=U ; \lambda=0.1$. The gray color indicate regions where the Dirac points of the low-energy states leave the bulk gap merging into the projected bulk bands. Here $\tilde{l}=l \sqrt{\frac{\Xi_{0}}{B}}, \tilde{U}=\frac{d U}{\sqrt{B \Xi_{0}}}$, and $\lambda=\frac{A^{2}}{4 B \Xi_{0}}$.

$\left|U_{1}+U_{2}\right| \gg \Xi_{0}$, or weak, $\left|U_{1}+U_{2}\right| \ll \Xi_{0}$, the energies are very small, $\left|E_{\varphi 0}\right|,\left|E_{\chi 0}\right| \ll \Xi_{0}$; therefore, one can obtain simple estimation of the characteristic momenta: $\operatorname{Re}\left(q_{1,2}\right)=$ $q_{0}=A / 2 B$ and $\operatorname{Im}\left(q_{1,2}\right)=w_{0}=q_{0} \sqrt{\lambda^{-1}-1}$. So, under in- creasing $|A|$ (or decreasing $B$ ) the low-energy states become stronger confined to the TI/NI interfaces. The decrease of $\lambda$ leads to more frequent oscillations of the EFs over the decay distance.
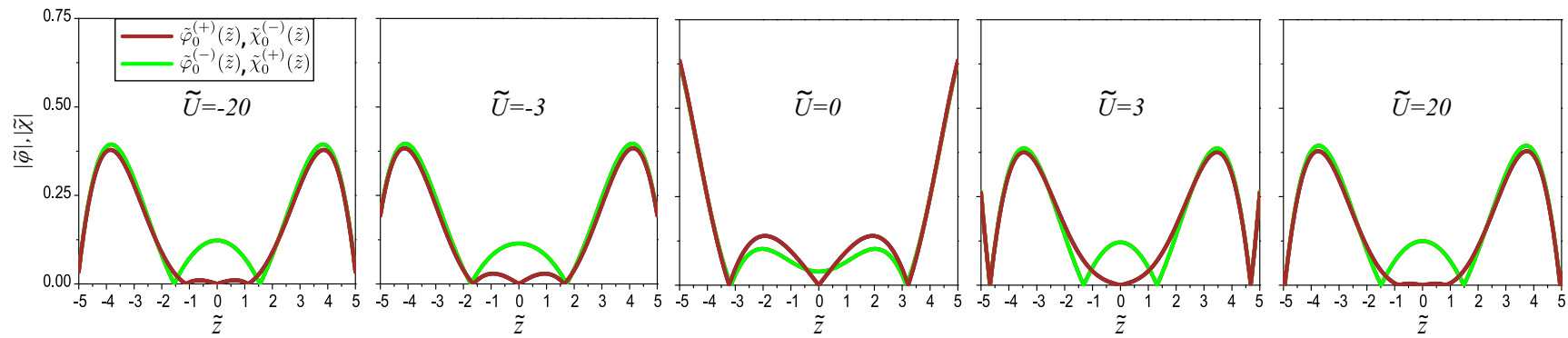

FIG. 2. Modules of space profiles for components of the bispinors $\varphi_{0}^{(\sigma)}$ and $\chi_{0}^{(\sigma)}$ in the TI film of thickness $2 \tilde{l}=10$ for several values for IP with $U_{1}=-U_{2}=U$ at $\lambda=0.25$. Here $\tilde{l}=l \sqrt{\frac{\Xi_{0}}{B}}, \tilde{U}=\frac{d U}{\sqrt{B \Xi_{0}}}$, and $\lambda=\frac{A^{2}}{4 B \Xi_{0}}$. 

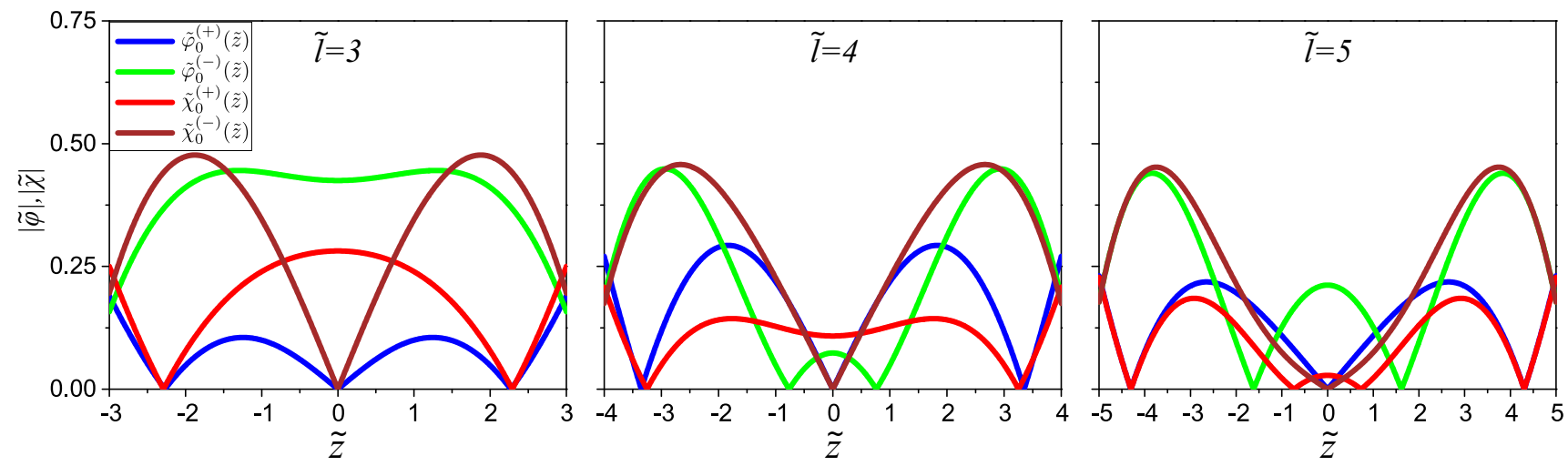

FIG. 3. Space dependence of envelope functions for $\tilde{U}=5$ and several values of thickness $\tilde{l}$ at $\lambda=0.25$ and $U=U_{1}=U_{2}$. Here $\tilde{l}=l \sqrt{\frac{\Xi_{0}}{B}}$, $\tilde{U}=\frac{d U}{\sqrt{B \Xi_{0}}}$, and $\lambda=\frac{A^{2}}{4 B \Xi_{0}}$.

Remarkably, as seen in Figs. 2 and 3, the EF behavior near the TI/NI interfaces and, in particular, the boundary amplitudes $\varphi_{0}^{\sigma}( \pm l)$ and $\chi_{0}^{\sigma}( \pm l)$ are directly determined by the IP strength and sign. As it is represented in Fig. 1, one can manipulate the positions of the energy levels $E_{\varphi 0}$ and $E_{\chi 0}$ through the IP matrix elements. This means that the in-gap states in the NI/TI/NI trilayer are essentially interface-specific states.

\section{EFFECTIVE 2D HAMILTONIAN OF THE 3D TI FILM}

We focus on the long-wavelength states with smallmomentum $\boldsymbol{\kappa}$ and low-energy $E_{\varphi, \chi}$. As seen in Fig. 1, there is a wide range of values of the model parameters, at which the low-energy states $(n=0)$ are clearly separated in energy from high-energy states $(|n| \geqslant 1)$; i.e., $\left|E_{\varphi, \chi 0}-E_{\varphi, \chi n}\right| \gtrsim$ $2 \Xi_{0} \sqrt{\lambda(1-\lambda)}$. This allows us (see also Refs. [14,40]) to project the complete set of the eigenstates of the problem Eqs. (7)-(9) onto a subspace spanned by a minimal orthogonal basis set composed of the four low-lying states at $\boldsymbol{\kappa}=$ $0, \varphi_{0}^{\sigma}(z)$, and $\chi_{0}^{\sigma}(z)$, and then construct the perturbation theory on this truncated basis in terms of a small value of $\mathbb{H}\left(\boldsymbol{\kappa},-i \partial_{z}\right)-\mathbb{H}\left(0,-i \partial_{z}\right)$ proportional to $\boldsymbol{\kappa}$. By applying the scheme $[14,40]$ to the NI/TI/NI trilayer, we derive the effective 2D Hamiltonian specific for a TI spacer, which can be written in the block-diagonal form:

$$
\begin{gathered}
\mathbb{H}_{\mathrm{eff}}(\boldsymbol{\kappa})=\epsilon_{0}(\boldsymbol{\kappa}) \mathbb{I}+\operatorname{diag}\left\{\mathcal{F}^{\Uparrow}(\boldsymbol{\kappa}), \mathcal{F}^{\Downarrow}(\boldsymbol{\kappa})\right\}, \\
\mathcal{F}^{\Uparrow}(\boldsymbol{\kappa})=\left(\begin{array}{cc}
\Delta(\kappa) & \mathcal{A} k_{-} \\
\mathcal{A}^{*} k_{+} & -\Delta(\kappa)
\end{array}\right), \\
\mathcal{F}^{\Downarrow}(\boldsymbol{\kappa})=\left(\begin{array}{cc}
-\Delta(\kappa) & -\mathcal{A}^{*} k_{-} \\
-\mathcal{A} k_{+} & \Delta(\kappa)
\end{array}\right) .
\end{gathered}
$$

The Hamiltonian Eqs. (11) and (12) is split into two parts defined by $\mathcal{F}^{\Uparrow}$ and $\mathcal{F}^{\Downarrow}$ with opposite projections of the pseudospin degree of freedom (symbolized with $\Uparrow$ $/ \Downarrow)$, which here is a good quantum number [14]. We use the following notations: $\epsilon_{0}(\kappa)=E_{0}-D \kappa^{2}, 2 E_{0}=E_{\varphi 0}+$ $E_{\chi 0}, 2 D=B_{\varphi}+B_{\chi}, \Delta(\kappa)=\Delta_{0}-b \kappa^{2}, 2 \Delta_{0}=E_{\varphi 0}-E_{\chi 0}$, $2 b=B_{\varphi}-B_{\chi}$, and $k_{ \pm}=k_{x} \pm i k_{y}$. These parameters are de- termined by the matrix elements: $B_{\varphi}=B \int_{-l}^{l} d z\left(\varphi_{0}^{\sigma}\right)^{\dagger} \sigma_{z} \varphi_{0}^{\sigma}$ and $B_{\chi}=B \int_{-l}^{l} d z\left(\chi_{0}^{\sigma}\right)^{\dagger} \sigma_{z} \chi_{0}^{\sigma}$ under the normalization requirement $\int_{-l}^{l} d z\left(\varphi_{0}^{\sigma}\right)^{\dagger} \sigma_{0} \varphi_{0}^{\sigma}=\int_{-l}^{l} d z\left(\chi_{0}^{\sigma}\right)^{\dagger} \sigma_{0} \chi_{0}^{\sigma}=1$. In turn, $A_{\varphi \chi}^{\sigma,-\sigma}=A \int_{-l}^{l} d z\left(\varphi_{0}^{\sigma}\right)^{\dagger} \sigma_{x} \chi_{0}^{-\sigma}=\mathcal{A}, A_{\chi \varphi}^{\sigma,-\sigma}=\left[A_{\varphi \chi}^{-\sigma, \sigma}\right]^{*}$, and $A_{\chi \varphi}^{\sigma,-\sigma}=-A_{\chi \varphi}^{-\sigma, \sigma}$. For the sake of clarity, below we restrict the variation of $E_{\varphi 0}$ and $E_{\chi 0}$ to the projected bulk bang gap, where the characteristic momenta of Eq. (10) are complex, $q_{1,2}(E)=q(E) \pm i w(E) ; q$ and $w$ are real. Yet, note that $|D|$ must be smaller than $|b|$, otherwise the gap disappears and further discussion is not relevant.

Importantly, in our approach the parameters $E_{0}, D, \Delta_{0}, b$, and $\mathcal{A}$ specifying the Hamiltonian $\mathbb{H}_{\text {eff }}$ depend not only on the characteristics of the 3D TI bulk spectrum, $\Xi_{0}, B, A$, but also on the film thickness, $2 l$, and the IP components, $U_{1,2}$. The corresponding analytical expressions can be obtained in terms of elementary functions, but they are highly cumbersome.

First, it is instructive to study how the electron structure of the film depends on $l$ and $U_{1,2}$ in some limiting cases. Let us consider the film with thickness that exceeds the EF variation scale, $\sim \exp (-2 q l) \ll 1$. When $\left|E_{\varphi, \chi 0}\right| \ll \Xi_{0} \sqrt{\lambda(1-\lambda)}$, the basis bispinors acquire the following form $(|z|<l)$ :

$$
\begin{gathered}
\varphi_{0}^{\sigma}=a_{\varphi}^{\sigma}\left(\begin{array}{c}
i \sum_{n= \pm 1} \psi(l+n z) \\
\sigma \operatorname{sgn}(A) \sum_{n= \pm 1} n \psi(l+n z)
\end{array}\right), \\
\chi_{0}^{\sigma}=a_{\chi}^{\sigma}\left(\begin{array}{c}
i \sum_{n= \pm 1} n \psi(l+n z) \\
\sigma \operatorname{sgn}(A) \sum_{n= \pm 1} \psi(l+n z)
\end{array}\right),
\end{gathered}
$$

where

$$
\psi(\zeta)=\sin \left(w_{0} \zeta+\gamma\right) \exp \left(-q_{0} \zeta\right)
$$

is a scale function of the in-gap bound states inherent to an semi-infinite 3D TI slab, which have been thoroughly described in Ref. [38]. In other words, EFs $\varphi_{0}^{\sigma}$ and $\chi_{0}^{\sigma}$ in Eq. (12) are approximated by the symmetric and antisymmetric combinations of the bound states coming from the opposite interfaces at $z= \pm l$. The characteristic momenta $q_{0}=q(E=$ $0)=|A| / 2 B$ and $w_{0}=w(E=0)=q_{0} \sqrt{\lambda^{-1}-1}$ are determined by the bulk spectrum parameters, while the phase shift $\gamma$ is dominated by the IP, $a_{\varphi, \chi}^{\sigma}$ is a normalization constant, 
and $k_{m}^{2}=w_{0}^{2}-q_{0}^{2}$. In the specific case of the "staggered" composition of the matrix elements, $U_{1}=-U_{2}=U$, when the IP does not break the particle-hole symmetry, the relation between $\gamma$ and $U$ may be represented explicitly for arbitrary strength of the IP: $\sin \gamma=\frac{\sqrt{1-\lambda}}{\sqrt{1-\lambda+\tilde{U}^{2}}}$ and $\cos \gamma=\frac{-\tilde{U}}{\sqrt{1-\lambda+\tilde{U}^{2}}}$. The EF magnitude at the interface is directly determined by the IP strength, $\left|\varphi_{0}^{\sigma}( \pm l)\right|^{2} \approx\left|\chi_{0}^{\sigma}( \pm l)\right|^{2} \approx \frac{q_{0}}{1+(\sqrt{\lambda}-\tilde{U})^{2}}$. The parameters of the effective Hamiltonian in the leading asymptotic term in the overlap $\sim \exp \left(-2 q_{0} l\right) \ll 1$ are given by

$$
\begin{gathered}
\Delta_{0}=8|a|^{2} B w_{0} \sin \left(2 w_{0} l+2 \gamma\right) \exp \left(-2 q_{0} l\right), \\
b=-4|a|^{2} B l \cos \left(2 w_{0} l+2 \gamma\right) \exp \left(-2 q_{0} l\right),
\end{gathered}
$$

where $|a|^{2} \equiv\left|a_{\varphi}^{\sigma}\right|^{2}=\left|a_{\chi}^{\sigma}\right|^{2}=\frac{q_{0}\left(1-\lambda+\tilde{U}^{2}\right)}{(1-\lambda)\left[1+(\sqrt{\lambda}-\tilde{U})^{2}\right]}, \epsilon_{0}(\kappa)=0$, and $A_{\varphi \chi}^{\sigma,-\sigma}=i \sigma|A|\left(a_{\varphi}^{\sigma}\right)^{*} a_{\chi}^{-\sigma} /|a|^{2}$.

If $U_{1} \neq-U_{2}$, the analytical estimations can be obtained to the leading order in $\left|U_{1,2}\right| \Xi_{0}^{-1}$ or $\left|U_{1,2}^{-1}\right| \Xi_{0}$. So, in the case of the strong IP, $\left|U_{1}^{-1}+U_{2}^{-1}\right| \ll \Xi_{0}^{-1}$, the EF Eq. (13) vanishes at the interface as $\varphi_{0}^{\sigma}( \pm l) \sim \chi_{0}^{\sigma}( \pm l) \sim \gamma \sim\left(U_{1}^{-1}+U_{2}^{-1}\right) \Xi_{0}$. Then one arrives at

$$
\begin{gathered}
E_{0}=-|A| B\left(q_{0}^{2}+w_{0}^{2}\right) d^{-1}\left(U_{1}^{-1}+U_{2}^{-1}\right), \\
D=-B E_{0} / \Xi_{0}, \\
\Delta_{0}=-2|A| \frac{q_{0}^{2}+w_{0}^{2}}{w_{0}} \sin \left(2 w_{0} l\right) \exp \left(-2 q_{0} l\right), \\
b=2|A| \frac{q_{0}^{2}+w_{0}^{2}}{w_{0}^{2}} l \cos \left(2 w_{0} l\right) \exp \left(-2 q_{0} l\right) .
\end{gathered}
$$

In turn, in the case of the weak IP, $\left|U_{1}+U_{2}\right| \ll \Xi_{0}$, the EF Eq. (13) reaches a maximal value at the interface since $2 \gamma \pm \pi \sim\left(U_{1}+U_{2}\right) \Xi_{0}^{-1}$. Then one obtains

$$
\begin{gathered}
E_{0}=2 q_{0} \frac{q_{0}^{2}+w_{0}^{2}}{2 q_{0}^{2}+w_{0}^{2}} d\left(U_{1}+U_{2}\right), \\
D=0, \\
\Delta_{0}=2|A| \frac{w_{0}\left(q_{0}^{2}+w_{0}^{2}\right)}{2 q_{0}^{2}+w_{0}^{2}} \sin \left(2 w_{0} l\right) \exp \left(-2 q_{0} l\right), \\
b=-2|A| \frac{q_{0}^{2}+w_{0}^{2}}{2 q_{0}^{2}+w_{0}^{2}} l \cos \left(2 w_{0} l\right) \exp \left(-2 q_{0} l\right) .
\end{gathered}
$$

Thus, we have derived the explicit thickness and IP dependencies of the parameters of the Hamiltonian $\mathbb{H}_{\text {eff }}$. One can observe that the IP affects significantly the low-energy states of the $3 \mathrm{D}$ TI film. The composition of the TI matrix elements and the IP strength regulate essentially the space behavior of the film states near the interfaces for the relatively thick film. This fact is clearly illustrated by the expression Eqs. (13) and (14) for the EF. The diagonal term $\Delta(\kappa)$ in effective Hamiltonian Eqs. (11) and (12) is determined by the hybridization gap in the $2 \mathrm{D}$ Brillouin zone center, $\Delta_{0}$, and the dispersion parameter, $b$, both of which are caused by the overlap of the states [see Eq. (13)] coming from the opposite interfaces. The diagonal term, $\Delta(\kappa)$, specifies the topological properties of the NI/TI/NI trilayer (as we will show in the next subsection). We find the important fact that the confinement characteristics $l$ and $U_{1,2}$ exert direct influence on the band structure parameters $\Delta_{0}$ and $b$. As seen in Eqs. (15) and (16), (19) and (20), (23) and (24), by varying the film thickness and/or the IP strength, one can repeatedly invert both the hybridization gap $2 \Delta_{0}$ and the dispersion parameter $b$, and thus cause the topological phase transition. The variation of both $\Delta_{0}$ and $b$ with the thickness is dominated by the bulk spectrum parameters via $q_{0}$ and $w_{0}$. Importantly, with the increasing film thickness the decay length of the diagonal term, $\Delta(\kappa)$, is longer than its oscillation period, e.g., $w_{0}=3 q_{0}$ at $\lambda=0.1$. The oscillating functions $\Delta_{0}(l)$ and $b(l)$ are shifted from each other by the quarter period. It should be noted that the parameters $\Delta_{0}$ and $b$ change sign when IP switches from the weak limit to the strong one. The term $E_{0}(\kappa)$ accounts for the particle-hole asymmetry generated by the IP with the composition $U_{1} \neq U_{2}$ in accordance with Eqs. (17) and (21).

As for the off-diagonal terms in Eq. (12), in the case $U_{1}=-U_{2}$, the magnitude $|\mathcal{A}|$ does not depend on $l$ and $U$ and takes the value of $2 \sqrt{\lambda}$. In the case $U_{1}=U_{2}$, the parameter $|\mathcal{A}|$ equals also to $2 \sqrt{\lambda}$ when the IP is either weak or strong. However, under the moderate values of the IP, the behavior of the off-diagonal terms becomes more complicated: the parameter $|\mathcal{A}|$ is smaller than $2 \sqrt{\lambda}$ and oscillates as a function of $l$ around some value. These details of the off-diagonal terms depending on the film thickness and IP are taken into account in our calculations.

Having analytically established the fact that the IP can affect the electron properties of the TI film in the NI/TI/NI trilayer, we further verify this result by numerical calculations for a wide region of $l$ and $U_{1,2}$. In Fig. 4, under moderate strength of the IP, the hybridization energy gap and the dispersion parameter are plotted versus the film thickness in the semilogarithmic scale that displays the damped oscillation pattern. This pattern shifts with the IP strength $U$ along the horizontal axis $l$.

The spectrum of the Hamiltonian Eqs. (11) and (12) consists of two branches expressed as

$$
E_{ \pm}(\kappa)=\epsilon_{0}(\kappa) \pm \sqrt{\Delta^{2}(\kappa)+|\mathcal{A}|^{2} \kappa^{2}} .
$$

The energy $E_{0}$ shifts the bands $E_{ \pm}(\kappa)$ with respect to the position of the Fermi level. When $D^{2}<b^{2}$, the energy gap between the electronlike band $E_{+}(\kappa)$ and the holelike band $E_{-}(\kappa)$ appears. The difference $E_{+}-E_{-}$at $\kappa=0$ is equal to $2\left|\Delta_{0}\right|$. If the product $b \Delta_{0}$ is positive and as large as $2 b \Delta_{0}>|\mathcal{A}|^{2}$, the spectrum Eq. (25) acquires the "camelback" shape so the true band gap (i.e., the minimum energy separation between the band branches) is not located in a center of the $2 \mathrm{D}$ Brillouin zone but on a circle of the radius $\kappa_{m}$. For example, at $\omega_{0}(\kappa)=0$, one has $\kappa_{m}=\sqrt{\frac{\Delta_{0}}{b}} \sqrt{1-2 \Lambda}$, and the true gap is $E_{+}\left(\kappa_{m}\right)-E_{-}\left(\kappa_{m}\right)=4\left|\Delta_{0}\right| \sqrt{\Lambda(1-\Lambda)}<2\left|\Delta_{0}\right|$; here the parameter $\Lambda=\frac{|\mathcal{A}|^{2}}{4 b \Delta_{0}}$ is used. However, in the case when $\left|U_{1}^{ \pm 1}+U_{2}^{ \pm 1}\right|<\Xi_{0}^{\mp 1}$ and $\exp \left(-2 q_{0} l\right)$, the value $b \Delta_{0}$ can be as small as $b \Delta_{0}<|\mathcal{A}|^{2}$, then the true gap opens at $\kappa=0$ and coincides with the value $E_{+}(0)-E_{-}(0)=2\left|\Delta_{0}\right|$. If the product $b \Delta_{0}$ is negative, the minimum excitation energy occurs always at $\kappa=0$ being equal to the hybridization gap $2\left|\Delta_{0}\right|$. As the parameters $\Delta_{0}$ and $b$ are the functions of $l$ and $U_{1,2}$, the spectrum peculiarities of the NI/TI/NI trilayer are governed 

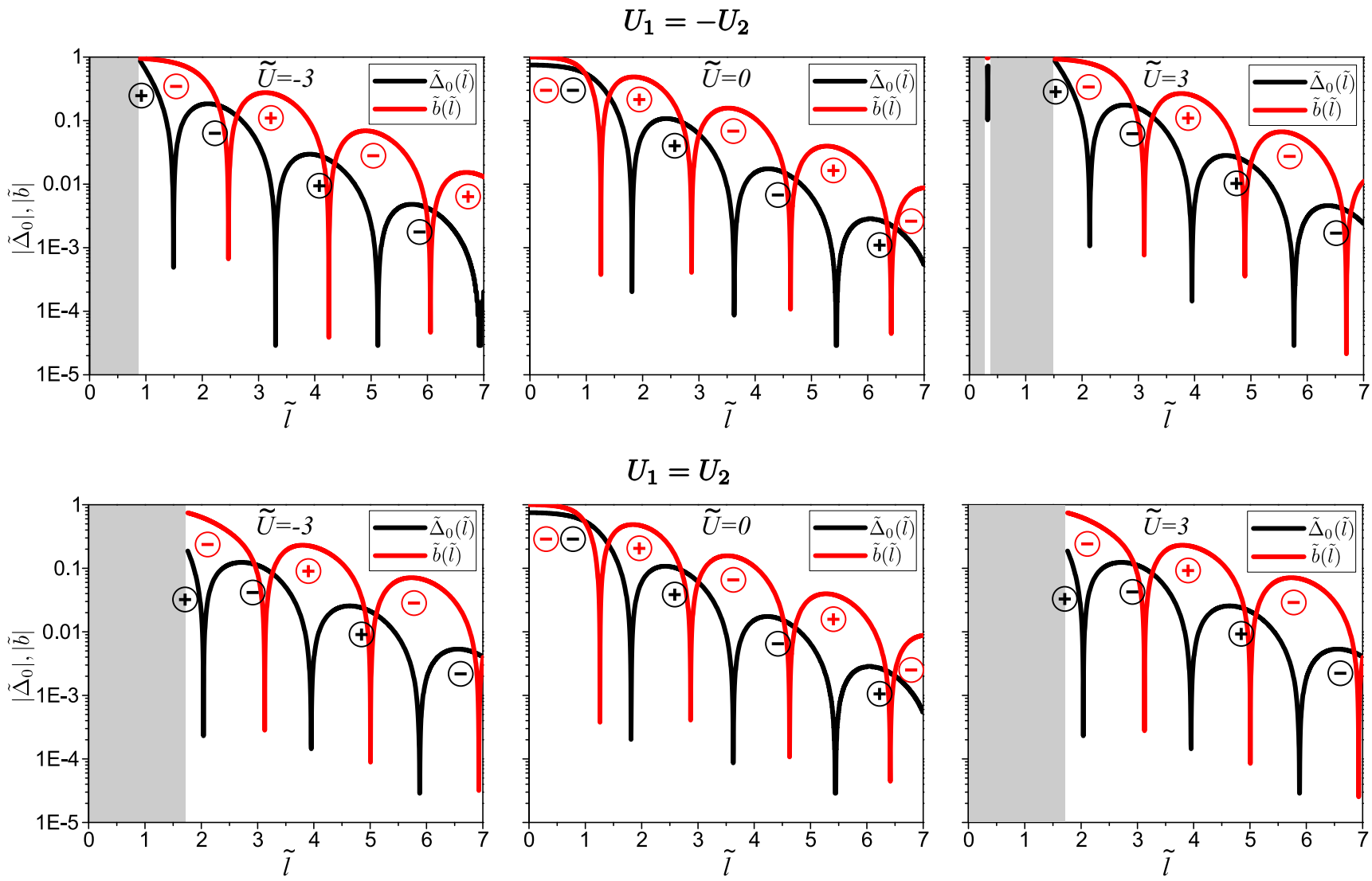

FIG. 4. Hybridization energy gap $2 \Delta_{0}$ (black lines) and dispersion parameter $b$ (red lines) in effective 2D model for the 3D TI film plotted as a function of the film thickness $2 l$ in the case of $U_{1}=-U_{2}=U$ (upper row) and $U_{1}=U_{2}=U$ (lower row) at $\tilde{U}=0, \pm 3$ and $\lambda=0.25$. The signs of these parameters are indicated in the figure. The logarithms of the energy gap $2 \Delta_{0}$ and dispersion parameter $b$ are plotted vs. $2 l$ to demonstrate that they decay exponentially and oscillate with phase shift. The gray color indicate regions where the low-energy states leave the bulk gap merging into the projected bulk states. Here $\tilde{\Delta}_{0}=\frac{\Delta_{0}}{\Xi_{0}}$ and $\tilde{b}=\frac{b}{B}$.

by both the TI film thickness and IP. As an example, in Fig. 5 we provide a series of the energy dispersions calculated for different film thicknesses at the fixed IP strength.

Note that in the limiting case $q_{0} l \rightarrow \infty$, when the overlap of EFs localized at/near the opposite interfaces of the film is negligible, the dispersion relation Eq. (25) acquires the gapless Dirac conelike form, $E_{ \pm}(\kappa)=\epsilon_{0}(\kappa) \pm|\mathcal{A}| \kappa$, where the crossing point is shifted in the energy to the $E_{0}$ level and the cone surface is slightly curved due to the term $-D \kappa^{2}$.

\section{PECULIARITIES OF THE INTRINSIC SPIN HALL RESPONSE AND TOPOLOGICAL PHASE DIAGRAM OF THE UNBOUNDED NI/TI/NI TRILAYER}

From the low-energy states of the TI film sandwiched by the NI slabs, we have derived the effective 2D Hamiltonian having a physically transparent and compact form to describe how the electron transport properties of the NI/TI/NI layer are determined by the interfacial and finite-size effects. According to the Chern insulator concept [44], the spin Hall response (topological or trivial) is controlled by the diagonal term $\Delta(\kappa)$. In the present approach, both components of $\Delta(\kappa)=\Delta_{0}-$ $b \kappa^{2}$, being interrelated, $\frac{b}{\Delta} \sim l \cot \left(2 w_{0} l\right)$, depend essentially on the thin thickness and IP: $\Delta_{0}\left(l, U_{1,2}\right)$ and $b\left(l, U_{1,2}\right)$ exhibit an oscillatory behavior as functions of $l$, while the value and sign of $U_{1,2}$ determine the phase of these oscillations [see Eqs. (15) and (16), (19) and (20), (23) and (24), and Fig. 4]. Moreover, in certain region of the trilayer characteristics $\left(l, U_{1,2}\right)$, the low-energy states are pushed into the projected bulk bands. These theoretical observations suggest a possibility to control the conductivity of the NI/TI/NI trilayer via variation of the TI spacer thickness and interface potential.

Proceeding to the transport properties analysis, one needs to point at some methodical aspects. As noted in Ref. [40], the dimensional reduction procedure from the 3D model Eqs. (1) and (2) to the effective 2D model Eqs. (11) and (12) is valid under the stipulation $|\mathcal{A}|^{2} \lesssim b \Delta_{0}$ (or $|\Lambda| \lesssim 1$ ). Furthermore, the 2D Hamiltonian Eqs. (11) and (12), derived from the perturbation theory to low orders in $\kappa$, is correct only in a small vicinity of the $2 \mathrm{D}$ Brillouin zone origin, $\left(|\mathcal{A}| \kappa,|b| \kappa^{2}\right) \ll\left|\Delta_{0}\right|$. Therefore, one should keep in mind that the description of the spin Hall response of the NI/TI/NI trilayer in terms of the Chern insulator [44] is restricted. The topological character of such $2 \mathrm{D}$ system is mathematically featured by the intrinsic spin Hall response, $\sigma^{S}=\sigma_{x y}^{\Uparrow}-\sigma_{x y}^{\Downarrow}$, where $\sigma_{x y}^{\Sigma}$ is associated with the corresponding block $\mathcal{F}^{\Sigma}(\kappa)$ in Eq. (12) and $\Sigma=\Uparrow$, $\Downarrow$. It is convenient to represent the response in integer multiples of $\frac{e^{2}}{h}$ via the Chern number $C^{\Sigma}$, i.e., $\sigma_{x y}^{\Sigma}=\frac{e^{2}}{h} C^{\Sigma}$ ( $h$ is the Planck constant, $e$ is the electron charge). Within the framework of the Chern insulator model [44], when the chemical potential 

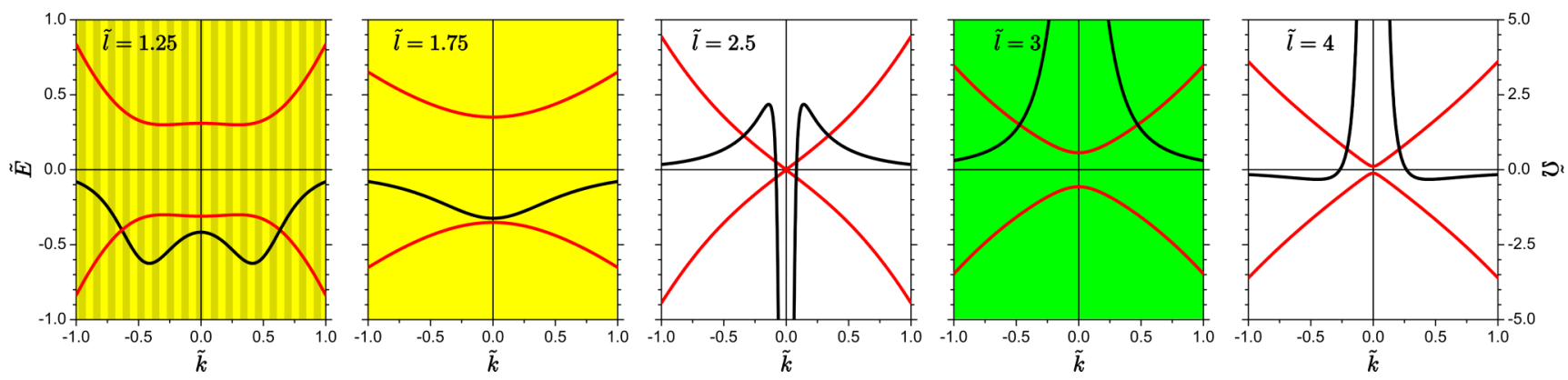

FIG. 5. Band spectra $E_{ \pm}(\kappa)$ (depicted by red lines) of the Hamiltonian Eqs. (11) and (12) and the corresponding Berry curvature distributions in momentum space $\Omega^{\Uparrow}(\kappa)$ (black lines) for different film thicknesses at the fixed IP strength $\tilde{U}=-1$. White background of the plot marks the trivial phase with $C^{S}=0$. Green color marks the QSHE phase with $C^{S}=1$, where $\Delta_{0}>0$ and $b>0$, while yellow marks the QSHE phase with $C^{S}=-1$, where $\Delta_{0}<0$ and $b<0$. The strip-shaded pattern indicates regions where $2 b \Delta_{0}>|\mathcal{A}|^{2}$ and the spectrum $E_{ \pm}(\kappa)$ has a "camelback" shape. Here $\tilde{E}=\frac{E}{\Xi_{0}}, \tilde{\Omega}=\Omega^{\Uparrow} \frac{\Xi_{0}}{B}$, and $\tilde{k}=k_{x, y} \sqrt{\frac{B}{\Xi_{0}}}$.

$\mu$ lies inside the energy gap, the factor $C^{\Sigma}$ is given by $C^{\Sigma}=\frac{1}{2 \pi} \int d \kappa \Omega^{\Sigma}(\kappa)$, where $\Omega^{\Sigma}(\kappa)$ is the Berry curvature, and the integral is performed over the first Brillouin zone. When the Hamiltonian is in the form of Eqs. (11) and (12), the Berry curvature is obtained as [14]

$$
\Omega^{\Uparrow}(\kappa)=\frac{|\mathcal{A}|^{2}\left(\Delta_{0}+b \kappa^{2}\right)}{2\left[\Delta^{2}(\kappa)+|\mathcal{A}|^{2} \kappa^{2}\right]^{\frac{3}{2}}},
$$

and $\Omega^{\Uparrow}=-\Omega^{\Downarrow}$. However, within our approach, the dependence $\Omega^{\Sigma}(\kappa)$ becomes ill-defined for momenta larger than $\kappa_{c} \approx \sqrt{\left|\frac{\Delta_{0}}{b}\right|}$. For example, in case of a strong/weak IP, using Eqs. (19), (20) and (23), (24), one can take the estimation $l \kappa_{c}^{2} \approx$ $\sqrt{\frac{\Xi_{0}}{B}}$. Nevertheless, if the electron states with momenta distant away from the center of the Brillouin zone are considered $a$ priori as inert in topological sense, the dependence Eq. (26) can be extended beyond $\kappa_{c}$ to capture principal features of the low energy transport.

The details of the Berry curvature distribution Eq. (26) vary remarkably depending on whether the parameters $\Delta_{0}$ and $b$ have the same or different signs. Let us assume provisionally that $\Delta_{0}>0$. If $\Lambda>\frac{2}{3}$ the function $\Omega^{\Uparrow}$ is positive and monotonic with the maximum at the origin $(\kappa=0)$. If $0<\Lambda<\frac{2}{3}$ the function $\Omega^{\Uparrow}(\kappa)$ is positive with the minimum at $\kappa=0$ and the maximum at $\kappa=\kappa_{0}$, where $\kappa_{0}^{2}=$ $\frac{\Delta_{0}}{2 b}[\sqrt{(\Lambda-1)(\Lambda-9)}-1-\Lambda]$. And if $\Lambda<1$ the function $\Omega^{\Uparrow}(\kappa)$ is sign-changing with the maximum at $\kappa=0$ and the minimum at $\kappa=\kappa_{0}$.

As the function $\Omega^{\Uparrow}(\kappa)$ decays quickly enough with increasing $\kappa$, the integral over the two-dimensional Brillouin zone can be replaced by the integral over the infinite 2D momentum space. This leads to the integer-valued factor $C^{\Uparrow}=-C^{\Downarrow}=$ $\frac{1}{2}\left[\operatorname{sgn}(b)+\operatorname{sgn}\left(\Delta_{0}\right)\right]$, provided that the chemical potential $\mu$ lies within the gap, so $|\mu|<2\left|\Delta_{0}\right| \sqrt{\Lambda(1-\Lambda)}$ at $0<\Lambda<\frac{1}{2}$ and otherwise $|\mu|<\left|\Delta_{0}\right|$. Thus, the spin Chern number [45] $C^{S}=\frac{1}{2}\left(C^{\Uparrow}-C^{\Downarrow}\right)$ equals 1 or -1 when $b \Delta_{0}>0$ and vanishes when $b \Delta_{0}<0$. Correspondingly, in a topological phase, $b \Delta_{0}>0$, the system is characterized by quantized plateaus in the spin transverse response as a function of $\mu$ with $\left|\sigma^{S}\right|=\frac{e^{2}}{h}$; while a trivial phase, $b \Delta_{0}<0$, is characterized by the zero spin response, $\sigma^{S}=0$.
In previous section we established that, by changing the IP and/or the TI film thickness, it is possible to change not only the value but also the sign of $\Delta_{0}$ and $b$. Having obtained the analytical expressions for $\Delta_{0}\left(l, U_{1,2}\right)$ and $b\left(l, U_{1,2}\right)$ of Eqs. (15) and (16), (19) and (20), (23) and (24) one can derive a simple formula for the spin Chern number in the trilayer:

$$
C^{S}=\frac{1}{2}\left\{\operatorname{sgn}\left[\sin \left(2 w_{0} l+2 \gamma\right)\right]-\operatorname{sgn}\left[\cos \left(2 w_{0} l+2 \gamma\right)\right]\right\} .
$$

Although this formula is strictly valid in the corresponding limiting situations, it reflects clearly the fact that both the interface conditions and the TI film thickness can drive the system through a quantum transition between the topological phase with $\left|C^{S}\right|=1$ and the trivial phase with $C^{S}=0$ and also explains how this transition occurs. In the trilayer with the relatively thick TI film, the parameters $\Delta_{0}\left(l, U_{1,2}\right)$ and $b\left(l, U_{1,2}\right)$ are the oscillating functions of $l$ with the period $l_{0}=\frac{\pi}{w_{0}}$ shifted from each other by the quarter period. Every sign alternation of either $\Delta_{0}\left(l, U_{1,2}\right)$ or $b\left(l, U_{1,2}\right)$ entails a stepped change in the spin Chern number. Hence, with varying thickness, the factor $C^{S}$ is described by the sequence $\ldots, 0,-1,0,1,0,-1,0,1, \ldots$ with the period $l_{0}$, so the topological phases with opposite sign of the spin Hall response, $\sigma^{S}=\frac{e^{2}}{h}$ and $\sigma^{S}=-\frac{e^{2}}{h}$, are separated from each other by the trivial phase with the zero response, $\sigma^{S}=0$. In other words, the variation of the TI film thickness by the quarter period, $\frac{l_{0}}{4}$, has to lead to a transition either from the topological phase to the trivial one or vice versa. On the other hand, the IP appears in Eq. (27) through the phase shift, $2 \gamma$. This means that the IP has a profound effect on the electron properties of the NI/TI/NI trilayer resulting in an alternation of the spin Chern number. So, the response $\sigma^{S}$ can switch between the quantized values $\pm \frac{e^{2}}{h}$ and 0 as the IP changes from the weak to strong limit.

To gain insight into the problem, we construct the topological phase diagram of the NI/TI/NI trilayer parameterized by $l$ and $U_{1,2}$ for the chemical potential positioned inside the gap. Phase transitions in our model can occur in two different ways: either by closing and reopening the hybridization gap $\Delta_{0}$ or by changing the sign of the term dispersion $b$. Respectively, the quantum phase transition between the nontrivial insulator, $b \Delta_{0}>0$, and the trivial band insulator, $b \Delta_{0}<0$, happens when the condition $\Delta_{0}\left(l, U_{1,2}\right)=0$ or $b\left(l, U_{1,2}\right)=0$ 
is satisfied. Figure 6 displays the phase diagram in terms of coordinates of the thickness and the IP strength for situations when $U_{1}=U_{2}=U$ (in this case the picture is symmetric with respect to the abscissa $U=0$ ) and $U_{1}=-U_{2}=U$, where $l$ and $U$ range from small to moderate values. One can readily see a stepwise behavior of the factor $C^{S}$ that changes discretely at the very phase boundaries determined by the equations $\Delta_{0}\left(l, U_{1,2}\right)=0$ and $b\left(l, U_{1,2}\right)=0$. Note that the quantum transition may be governed by both the TI film thickness and IP. Thus, looking at Fig. 6 one can propose two important for practical purposes routes to convert the trivial band insulator phase to the topological one and vice versa in the NI/TI/NI trilayers: either the variation of $U_{1,2}$, for example, by tuning the NI plates, at given $l$ or the variation of $l$ at given $U_{1,2}$. As seen in the phase diagram, both in the former case and in the latter case, the system can experience the quantum phase transition several times with increasing $l$ and $|U|$.

The expansion coefficients of the kp model for centrosymmetric TIs can be directly derived from $a b$ initio eigenfunctions of the bulk crystals at the $\Gamma$ point [46]. Here, taking into account the numerical results of Ref. [46], in the simplified Hamiltonian of Eq. (2) we adopt the following qualitative approximation for the band structure parameters: $|A|=0.2 \mathrm{eV}$ $\mathrm{nm}, B=0.5 \mathrm{eV} \mathrm{nm}^{2}$, and $\Xi_{0}=0.2 \mathrm{eV}$. Then one can obtain rough estimates: $\lambda=0.1, q_{0}=0.2 \mathrm{~nm}^{-1}, w_{0}=0.6 \mathrm{~nm}^{-1}$, and $l_{0}=5.2 \mathrm{~nm}$. At the fixed IP value and the enough thick TI film, the distance between neighboring phase boundaries in the diagrams in Fig. 6 is about $\frac{l_{0}}{4}=1.3 \mathrm{~nm}$. This means that a change of the TI film thickness in a few nanometers can trigger the topological phase transition.

The IP matrix elements can be intuitively associated with the relative energy offsets between the relevant band edges of TI and NI. From this viewpoint, the comparison between the analytic results and the data of the electron band structure calculations carried out within the density functional theory for some concrete interfaces provides the estimation of the IP components [38]: $U_{1} \simeq 0.2 \mathrm{eV}$ and $U_{2} \simeq 0 \mathrm{eV}$ for $\mathrm{GeBi}_{2} \mathrm{Te}_{4} / \mathrm{Bi}_{2} \mathrm{Te}_{2} \mathrm{~S} ; U_{1} \simeq-0.2 \mathrm{eV}$ and $U_{2} \simeq-0.4 \mathrm{eV}$ for $\mathrm{Sb}_{2} \mathrm{Te}_{2} \mathrm{~S} / \mathrm{Sb}_{2} \mathrm{Te}_{2} \mathrm{Se} ; U_{1} \simeq-0.4 \mathrm{eV}$ and $U_{2} \simeq-0.6 \mathrm{eV}$ for $\mathrm{Bi}_{2} \mathrm{Te}_{2} \mathrm{~S} / \mathrm{GeBi}_{2} \mathrm{Te}_{4}$. If one uses the band structure parameters $B=0.5 \mathrm{eV} \mathrm{nm}{ }^{2}$ and $\Xi_{0}=0.2 \mathrm{eV}$ and the length $d \simeq 1 \mathrm{~nm}$, the dimensionless strength can range from 0 to moderate values of the order of $|\tilde{U}| \simeq 2$. According to the diagrams in Fig. 6 , such values of the IP strength are sufficient to drive the TI/NI trilayer through the topological transition.

By varying the TI film thickness in the trilayer, we illustrate in Fig. 5 how the distribution of the Berry curvature in momentum space (together with the corresponding energy spectrum) changes under transition between topologically different phases. The red crosses in Fig. 6 indicate the points in the phase plane $(l, U)$, at which the dependencies $E_{ \pm}(\kappa)$ and $\Omega^{\Uparrow}(\kappa)$ are depicted in Fig. 5. While the band dispersions of the normal and inverted types are rather similar, the Berry curvature distributions in the topologically different phases are distinctly different.

We must also say that our approach does not reproduce the phase diagram correctly enough in close vicinity of the phase boundaries $\Delta_{0}\left(l, U_{1,2}\right)=0$ and $b\left(l, U_{1,2}\right)=0$. This is because the perturbative procedure to derive the $2 \mathrm{D}$ Hamiltonian Eqs. (11) and (12) fails when $|\mathcal{A}|^{2} \gtrsim\left|b \Delta_{0}\right|$.

\section{EDGE ELECTRON STATES AND QSHE IN THE BOUNDED NI/TI/NI TRILAYER}

In the previous section we have analyzed evolution of the topological insulating phase in the NI/TI/NI trilayer unbounded in the interface plane depending on the IP strength and the TI film thickness. The integer-quantized spin Hall response of this system proportional to $\sigma^{S}$ has been attributed to the spin Chern number $C^{S}$. The number $C^{S}$ is rather a mathematical feature inherent to the model Hamiltonian Eqs. (11) and (12) that is defined on the infinite plane. In realistic situations, the TI film is surrounded by other trivial media (material or vacuum), and therefore it is relevant to address the problem of what is a manifestation of the spin Hall response of the NI/TI/NI trilayer bounded in the interface plane $(x, y)$. When the TI film interior is insulating, the electronic transport at low temperature is dominated by the electron states at the film edges at $\Upsilon(x, y)=0$. It is usually believed $[9,10]$ that a nontrivial bulk topological invariant entails the existence of the Dirac-like helical state at the boundary with a trivial material. Within the kp scheme, one usually imposes the open boundary conditions on the TI wave function [14,30-32], which provides the guides to the assertion that the topological and nontopological phases are distinguished from each other by the presence and absence of the the Dirac-like states, respectively. This picture allows one to associate the spin Hall response, $\sigma^{S}$, with the spin transverse conductivity due to the edge bound states. In particular, the boundaries of the 2D TI are thought to host the $1 \mathrm{D}$ edge metallic channels which contribute to QSHE [9,10]. However, such simple boundary condition is highly rough treatment of complex physical phenomena at the edges in the TI/NI heterostructures. In this section, incorporating the phenomenological edge potential, $\mathrm{EP}, t \mathbb{V} \delta[\Upsilon(x, y)]$, we explore how the helical edge states in the TI film are affected by the edges. Using the generalized boundary conditions of the natural type, we study the role of EP in the model Eqs. (11) and (12) in a restricted geometry. Clarifying the existence condition of the edge states depending on the EP strength allows us to shed new light on the QSHE in the NI/TI/NI trilayer.

Here, we consider the film occupying the half-plane $x>$ 0 and bounded by the edge meeting a topologically trivial medium along the line $x=0$, i.e., $\Upsilon(x, y)=x$. Carrying out analytic calculations, the 2D Hamiltonian Eqs. (11) and (12) with additional modification at the edge will be used. The equations for the EF spinor, $\eta^{\Sigma}=\left(\eta_{1}^{\Sigma}, \eta_{2}^{\Sigma}\right)^{\text {tr }}$, in the $(x, y)$ plane read

$$
\mathcal{F}^{\Sigma}\left(-i \partial_{x}, k_{y}\right) \eta^{\Sigma}(x)=\mathcal{I} \varepsilon^{\Sigma}\left(k_{y}\right) \eta^{\Sigma}(x),
$$

where $k_{y}$ remains as a good quantum number, $\Sigma=\Uparrow / \Downarrow$. For simplicity, the topologically unessential term $\sim \epsilon_{0}(\kappa)$ in Eq. (11) is omitted. We imply that the EF decays far from the edge, $\eta^{\Sigma}\left(x \rightarrow \infty, k_{y}\right) \rightarrow 0$ and the natural boundary conditions are imposed on the EF at the edge,

$$
\left.i \frac{\partial \mathcal{F}^{\Sigma}\left(-i \partial_{x}, k_{y}\right)}{\partial\left(-i \partial_{x}\right)} \eta^{\Sigma}(x)\right|_{x=0}=2 t \mathcal{U} \eta^{\Sigma}(x=0),
$$

where $\mathcal{U}=\operatorname{diag}\{u, u\}$ is the EP matrix. EP acts on electrons near the TI film edge in a very narrow region, $\sim t$, of the order of an unit cell. We adopt a local approximation for EP, $\mathcal{U}(x) \rightarrow$ 

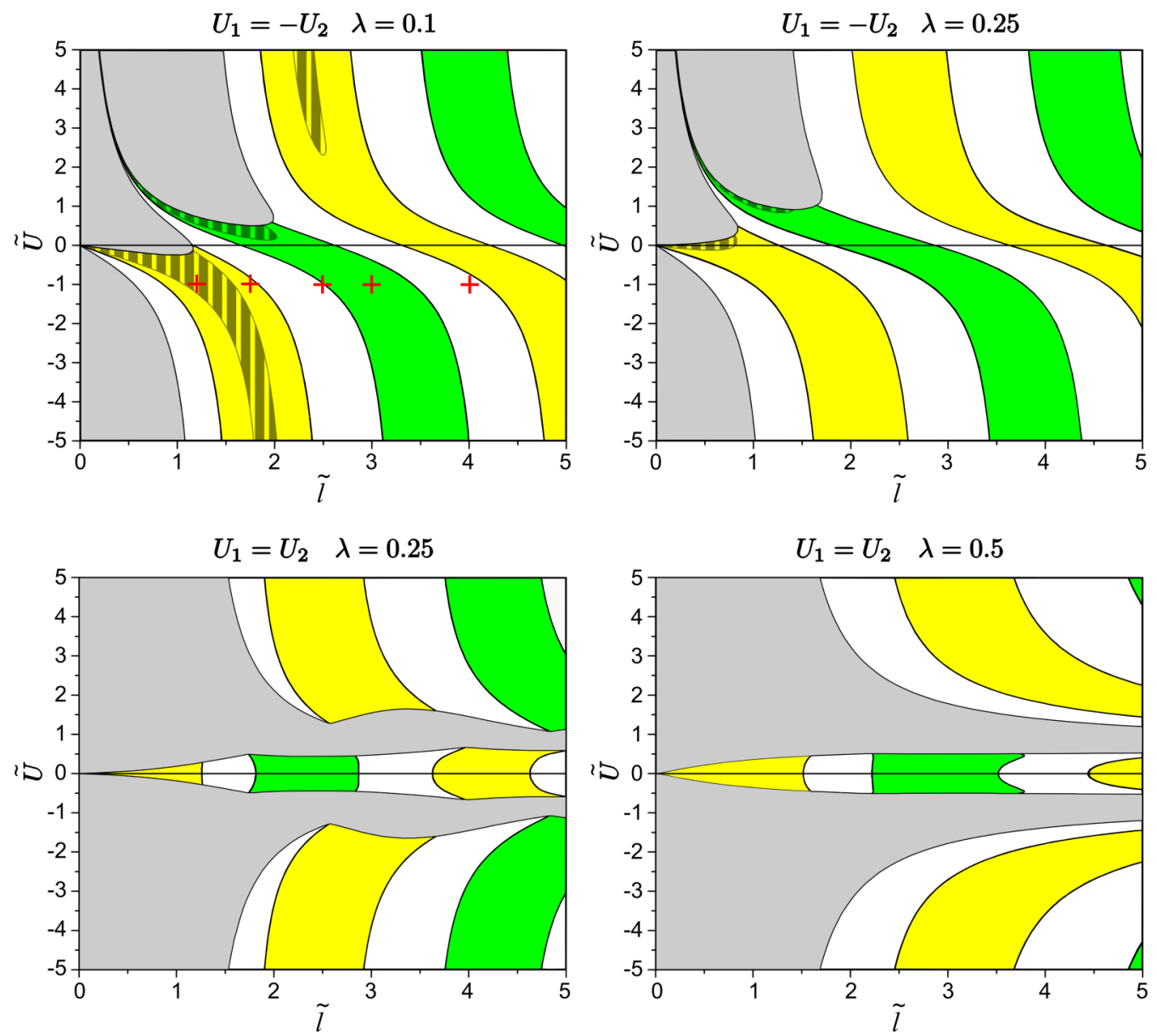

FIG. 6. Topological phase diagrams of the NI/TI/NI trilayer in the coordinates the IP strength vs. the film thickness when the chemical potential is inside the gap. Upper/lower row of the plots correspond to the situation when IP is arranged so $U_{1}=-U_{2}=U / U_{1}=U_{2}=U$. One can observe topologically distinct insulating phases characterized by the integer spin Chern numbers and residing in the corresponding domains. White domains mark the trivial phase with $C^{S}=0$. Green domains mark the QSHE phase with $C^{S}=1$, where $\Delta_{0}>0$ and $b>0$, while yellow domains mark the QSHE phase with $C^{S}=-1$, where $\Delta_{0}<0$ and $b<0$. Thick black curved lines distinguishing different phase domains are given by the equations $\Delta_{0}\left(l, U_{1,2}\right)=0$ and $b\left(l, U_{1,2}\right)=0$. The gray color indicates regions where the low-energy states leave the bulk gap merging into the projected bulk states. In the upper row, the strip-shaded areas show the $(U, l)$ regions where the spectrum $E_{ \pm}(\kappa)$ has a "camelback" shape. Here $\tilde{l}=l \sqrt{\frac{\Xi_{0}}{B}}, \tilde{U}=\frac{d U}{\sqrt{B \Xi_{0}}}$, and $\lambda=\frac{A^{2}}{4 B \Xi_{0}}$.

$\mathcal{U} \delta(x)$, because the scale $\sim t$ is significantly smaller than the EF spatial variation in the $(x, y)$ plane. This simplification is involved to study the 1D edge states by analogy with the case of IP, which has been used to describe the $2 \mathrm{D}$ bound states near the 3D TI surface [38]. For the NI/TI/NI trilayer, one can construct EPs differing in type and strength, for instance, by cutting the TI film crystal lattice along different crystal directions in the interface plane. Of course, either the existence or absence of the edge states in the NI/TI/NI trilayer at the given TI film thickness and IP is connected with the spin Chern number $C^{S}$, but questions on how this connection is realized and how the characteristics of these states are related with the boundary conditions containing EP have not been elucidated yet in the literature.

One can expect that the topological insulating phase with $\left|C^{S}\right|=1$, which covers the whole interior of the thin TI film, manifests itself through 1D conducting channels associated with the in-gap edge states at the side boundary, i.e., at $x=0$ in the $\mathbf{y}$ direction. Given $b \Delta_{0}>0$, in the case of both the strong
EP, $|\tilde{u}| \gg 1$, and the weak EP, $|\tilde{u}| \ll 1$, the energy levels of these states are pushed to the middle of the hybridization gap, $\left|\frac{\varepsilon^{\Sigma}}{\Delta_{0}}\right| \ll 1\left(\varepsilon^{\Sigma} \rightarrow 0\right)$, where $\tilde{u}=\frac{t u}{\sqrt{\left|b \Delta_{0}\right|}}$ is the dimensionless EP strength. Using this fact, after some calculations, one can find the analytic solution of Eqs. (28) and (29) in mathematically limiting situations. To be specific, let us assume that $0<\Lambda<$ 1. To the first order in $k_{y}$, the edge-state energy spectrum is presented by the Dirac dispersion relation slightly shifted with respect to the middle of the gap:

$$
\varepsilon^{\Sigma}\left(k_{y}\right)=\varepsilon_{0}+\operatorname{sgn}(b \Sigma)|\mathcal{A}| k_{y},
$$

where the Dirac point energy is $\varepsilon_{0}=-\frac{4\left|\Delta_{0}\right| \sqrt{\Lambda}}{\widetilde{u}}$ at $|\widetilde{u}| \gg 1$ and $\varepsilon_{0}=\frac{4\left|\Delta_{0}\right| \sqrt{\Lambda} \tilde{u}}{1+\Lambda}$ at $|\widetilde{u}| \ll 1$. Here and further the sign $\operatorname{sgn}(\Sigma)$ relates to the pseudospin index as $\operatorname{sgn}(\Sigma=\Uparrow)=+1$ and $\operatorname{sgn}(\Sigma=\Downarrow)=-1$. Quasiparticles in the edge states with opposite pseudospin projections, $\Uparrow$ and $\Downarrow$, propagate in opposite directions. The corresponding EFs satisfying the 

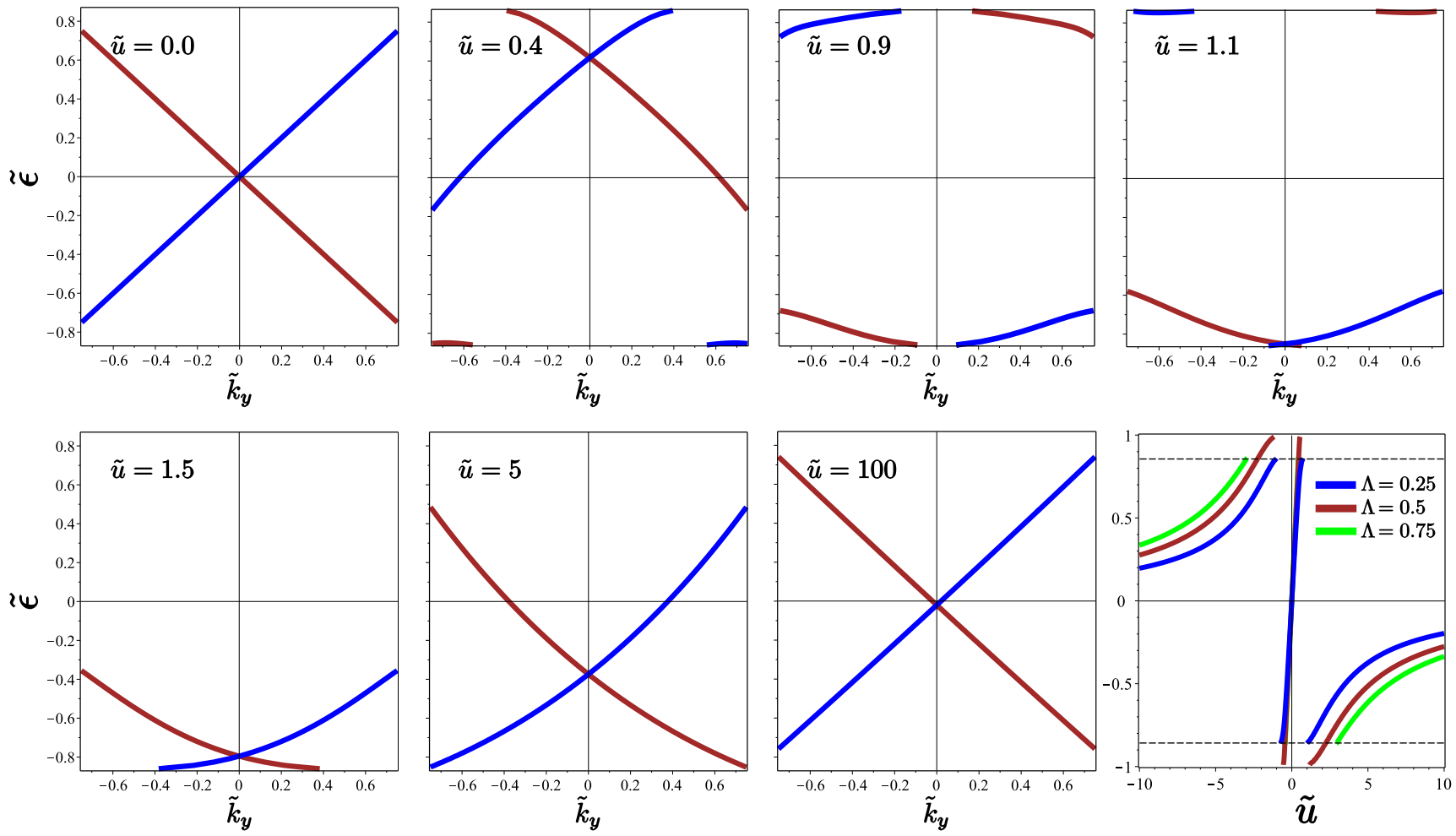

FIG. 7. Dispersion of the edge states depending on the EP strength in the QSHE regime. Blue/brown lines indicate the pseudospin polarization $\Uparrow / \Downarrow, \Lambda=0.25$. Last panel: The dependence of the Dirac point energy on the EP strength for several values of $\Lambda$, the dashed lines mark the energy gap for $\Lambda=0.25$ and $\Lambda=0.75$.

boundary condition Eq. (29) are given by

$$
\begin{aligned}
\eta_{1}^{\Sigma}(x)=\eta_{0} & \exp \left(-p_{0} x\right)\left[\left(1-\operatorname{sgn}(b \Sigma) \frac{\sqrt{\Lambda}}{\widetilde{u}}\right) \sin \left(s_{0} l\right)\right. \\
\left.-\operatorname{sgn}(b \Sigma) \frac{\sqrt{1-\Lambda}}{\widetilde{u}} \cos \left(s_{0} l\right)\right], & \\
\eta_{2}^{\Sigma}(x)= & i e^{i \phi} \operatorname{sgn}(b \Sigma) \eta_{0} \exp \left(-p_{0} x\right) \\
\times & {\left[\left(1+\operatorname{sgn}(b \Sigma) \frac{\sqrt{\Lambda}}{\widetilde{u}}\right) \sin \left(s_{0} l\right)\right.} \\
+ & \left.\operatorname{sgn}(b \Sigma) \frac{\sqrt{1-\Lambda}}{\widetilde{u}} \cos \left(s_{0} l\right)\right],
\end{aligned}
$$

for the strong EP;

$$
\begin{aligned}
& \eta_{1}^{\Sigma}(x)= \eta_{0} \exp \left(-p_{0} x\right)\left[\left(1+\operatorname{sgn}(b \Sigma) \frac{\tilde{u}}{1+\Lambda}\right) \cos \left(s_{0} l\right)\right. \\
&\left.-\operatorname{sgn}(b \Sigma) \frac{\tilde{u} \sqrt{1-\Lambda}}{1+\Lambda} \sin \left(s_{0} l\right)\right] \\
& \eta_{2}^{\Sigma}(x)= i e^{i \phi} \operatorname{sgn}(b \Sigma) \eta_{0} \exp \left(-p_{0} x\right) \\
& \times {\left[\left(1-\operatorname{sgn}(b \Sigma) \frac{\tilde{u}}{1+\Lambda}\right) \cos \left(s_{0} l\right)\right.} \\
&+\left.\operatorname{sgn}(b \Sigma) \frac{\tilde{u} \sqrt{1-\Lambda}}{1+\Lambda} \sin \left(s_{0} l\right)\right]
\end{aligned}
$$

for the weak EP. Here $\eta_{0}$ is the normalization constant. The edge states propagate along the $\mathbf{y}$ direction as plane waves, decaying away from the edge into the TI film interior region. The characteristic decay and oscillation lengths, which are inversely proportional to the momenta $p_{0}=\frac{|\mathcal{A}|}{2|b|}$ and $s_{0}=$ $p_{0} \sqrt{\Lambda^{-1}-1}$, respectively, are determined by the parameters of the plane Hamiltonian Eqs. (11) and (12). The oscillation phase is subjected to the boundary condition of Eq. (29), so the EF profile is very small at the edge $(x=0)$ when $|\widetilde{u}| \gg 1$, Eqs. (31) and (32), and peaked closely to the edge when $|\widetilde{u}| \ll$ 1, Eqs. (33) and (34). Curiously enough, the profile of the $\Uparrow$-polarized channel does not coincide with that $\Downarrow$-polarized channel, $\left|\eta_{1,2}^{\Uparrow}(x)\right| \neq\left|\eta_{1,2}^{\Downarrow}(x)\right|$, owing to the finite value of EP. Note also that the pseudospin-polarized channel flips its propagation direction as the parameter $\Delta_{0}$ (or $b$ ) changes sign.

Figure 7 demonstrates the modification of the band structure of the edge states with the varying EP. One can see in plots corresponding to $\widetilde{u}=0$ and $|\widetilde{u}| \gg 1$ that the nontrivial edge states connecting the $2 \mathrm{D}$ "valence" and "conduction" bands $E_{ \pm}(\kappa)$, Eq. (25), cross the hybridization gap. The appearance of such states with almost linear energy-momentum relation and the Dirac point placed within the hybridization gap, $\left|\varepsilon_{0}\right|<2\left|\Delta_{0}\right| \sqrt{\Lambda(1-\Lambda)}$, is consistent with the calculated Chern number $\left|C^{S}\right|=1$, confirming the nontrivial topological nature of the system. However, when the EP strength has moderate value, $|\widetilde{u}| \approx 1$, the Dirac-like dispersion is distorted. It is noteworthy that there is certain interval of the EP strength, $\widetilde{u}_{+}(\Lambda)>|\widetilde{u}|>\widetilde{u}_{-}(\Lambda)$, where the Dirac point merges into the $2 \mathrm{D}$ band continuum of Eq. (25), i.e., $\left|\varepsilon_{0}\right|>2\left|\Delta_{0}\right| \sqrt{\Lambda(1-\Lambda)}$; see the last panel in Fig. 7.

Let us analyze the trivial phase situation when $b \Delta_{0}<0$; i.e., $\Lambda<0$. First of all, note that in the cases of $|\widetilde{u}| \gg 1$ 

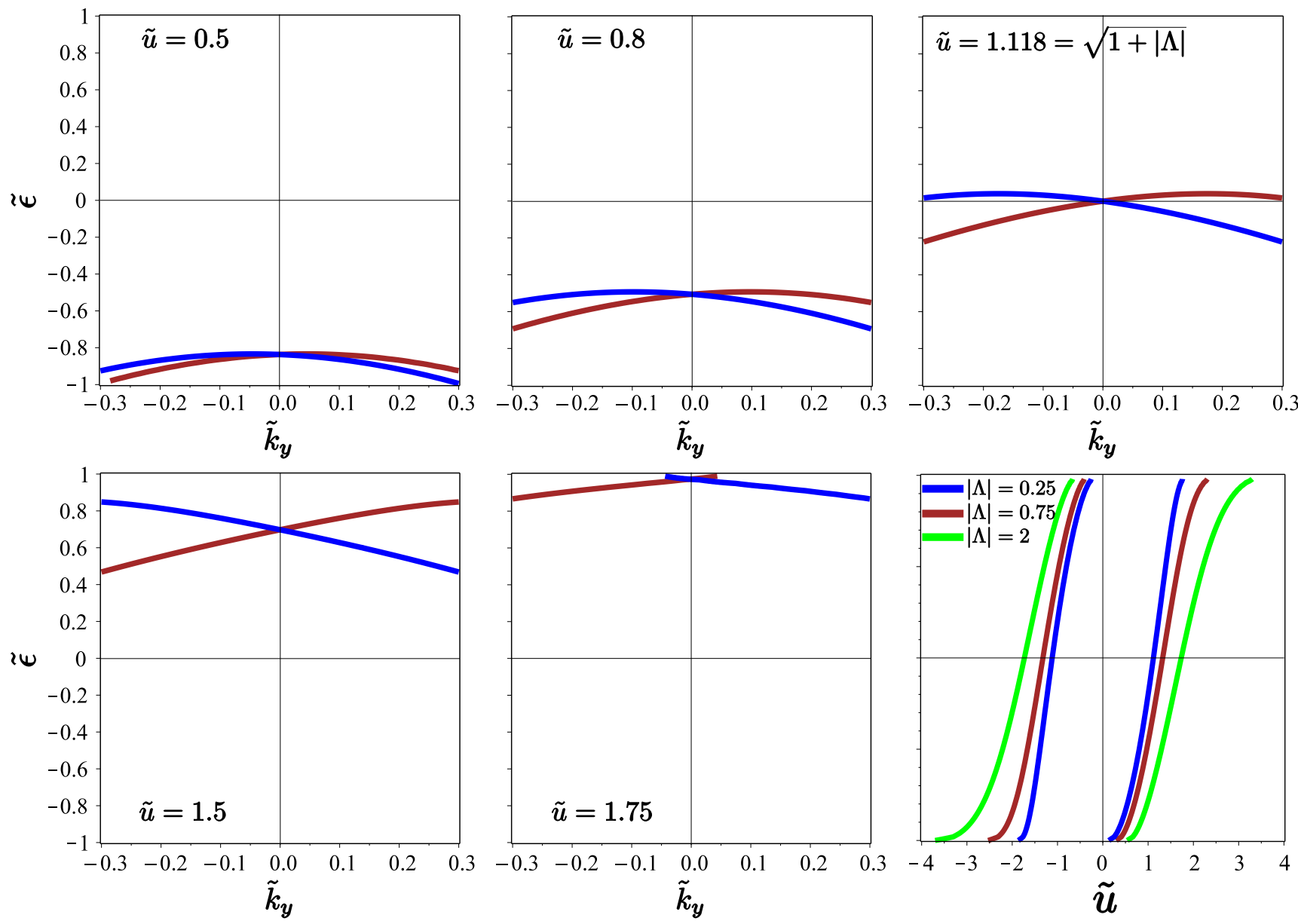

FIG. 8. Dispersion of the edge state depending on the EP strength in the trivial insulator regime. Blue/brown lines indicate the pseudospin polarization $\Uparrow / \Downarrow,|\Lambda|=0.25$. Last panel: The dependence of the Dirac point energy on the EP strength for several values of $\Lambda$.

and $|\tilde{u}| \ll 1$, Eqs. (28) and (29) have only a trivial solution, $\eta^{\Sigma}(x)=0$. In other words, at $C^{S}=0$ bound in-gap states are absent. Let us examine what happens in the case of EP of the moderate strength, $|\widetilde{u}| \approx 1$. It turns out that, for the small energy $\varepsilon^{\Sigma}\left(k_{y}\right)\left(\left|\varepsilon^{\Sigma}\left(k_{y}\right)\right| \ll\left|\Delta_{0}\right|\right)$ and momentum $k_{y}$, one can capture nontrivial solution of Eqs. (28) and (29) for the EP strength close to $|\widetilde{u}|=\sqrt{1+|\Lambda|}$. This solution is characterized by the Dirac-like dispersion:

$$
\varepsilon^{\Sigma}\left(k_{y}\right)=\varepsilon_{0}-\operatorname{sgn}(\Sigma) \operatorname{sgn}(b) \frac{\sqrt{|\Lambda|}}{\sqrt{1+|\Lambda|}}|\mathcal{A}| k_{y},
$$

where the Dirac point energy is $\varepsilon_{0}=-\operatorname{sgn}(u)\left|\Delta_{0}\right|\left(1-\frac{\widetilde{u}^{2}}{1+|\Lambda|}\right)$, and the Dirac velocity is renormalized with respect to the effective in-plane velocity. When $b \Delta_{0}<0$ and $\tilde{u}=$ $\operatorname{sgn}(b) \sqrt{1+|\Lambda|}$, we derive the simple expression for the corresponding EFs profiles:

$$
\begin{array}{r}
\eta_{1}^{\Sigma}(x)=\eta_{0}\left[\exp \left(-p_{1} x\right)+\operatorname{sgn}(b \Sigma) \exp \left(-p_{2} x\right)\right], \\
\eta_{2}^{\Sigma}(x)=\eta_{0} i e^{i \phi}\left[\exp \left(-p_{1} x\right)-\operatorname{sgn}(b \Sigma) \exp \left(-p_{2} x\right)\right] .
\end{array}
$$

A similar expression can be obtained when $\tilde{u}=$ $-\operatorname{sgn}(b) \sqrt{1+|\Lambda|}$. The penetration depths are determined by the smaller momentum of $p_{1,2}=\sqrt{\frac{\left|\Delta_{0}\right|}{|b|}}[\sqrt{1+|\Lambda|} \pm \sqrt{|\Lambda|}]$.
It is clearly seen that these EFs are mainly localized at the film edge.

Further, to acquire more quantitative knowledge of the existence of the in-gap edge states in the trivial insulating phase, we perform the numerical calculations. Figure 8 shows the evolution of the band structure of the edge states under the varying EP. As seen in the last panel in Fig. 8, the Dirac point of the edge state stays inside the hybridization gap when EP strength is within a certain narrow interval around the value of $|\tilde{u}|=\sqrt{1+|\Lambda|}$. Beyond this interval, the Dirac point merges into the $2 \mathrm{D}$ band continuum.

It is important to understand how the edge states described above contribute to QSHE in the NI/TI/NI trilayer. In the band structure theory of solids, the Chern number $C^{\Sigma}$ is formally introduced as an integral of the Berry phase curvature. This topological parameter is proportional to the static currentcurrent correlator of fermion on the infinite plane, which is calculated usually by means of the Kubo formula. In practice, the current-current correlator as a transverse response of the system to external electric field could be a measurable quantity in transport experiments on finite-size samples. For instance, the NI/TI/NI trilayer cut out in a shape of a ribbon of finite width in the $\mathbf{x}$ direction (the Hall bar geometry) can be utilized as a concrete experimental sample. In such a heterostructure sample, a quantization of spin transport is realized due to the 
1D helical edge states, which occur at side boundaries of the ribbon-shaped TI thin film and reside inside the hybridization gap $[9,10]$.

A pair of the counterpropagating edge states with opposite pseudospin polarization occurring at each edge of the ribbon contributes to QSHE under the stipulation that the chemical potential $\mu$ (fixed inside the hybridization gap) intersects the spectrum branches of the edge states on a linear segment near the Dirac point. In the NI/TI/NI trilayer, the gate voltage applied to the NI plates can provide a change in the chemical potential position inside the hybridization gap. The electric current, $I_{y}$, flowing in the $\mathbf{y}$ direction along the sample, due to the spin-momentum locking inherent to the edge states, induces a nonequilibrium spin accumulation at the opposite edges. The current flowing along the ribbon edges is the sum over all occupied states: $I_{y}^{\Sigma}=e \int \frac{d k_{y}}{2 \pi} v^{\Sigma}\left(k_{y}\right) n\left[\varepsilon^{\Sigma}\left(k_{y}\right)\right]$, where $v^{\Sigma}\left(k_{y}\right)=\frac{1}{\hbar} \frac{\partial \varepsilon^{\Sigma}\left(k_{y}\right)}{\partial k_{y}}$ is the group velocity, $n\left[\varepsilon^{\Sigma}\left(k_{y}\right)\right]$ is the occupation probability (the Fermi distribution function) of the $k_{y}$ th mode with the pseudospin polarization $\Sigma=\Uparrow, \Downarrow$, the full current is given by $I_{y}=I_{y}^{\Uparrow}+I_{y}^{\Downarrow}$. The cancellation of the group velocity and density of states, $\sim \frac{\partial k_{y}}{\partial \varepsilon^{\Sigma}}$, which usually takes place in a 1D system, leads to the relation $I_{y}^{\Sigma}=\frac{e}{h}\left(\mu_{R}^{\Sigma}-\mu_{L}^{\Sigma}\right) \operatorname{sgn}(\Sigma)$, where $\mu_{R / L}^{\Sigma}$ is the spin-dependent electrochemical potential at the right/left edge of the ribbon. In the ballistic regime, measuring a voltage between the edges as the imbalance between chemical potentials of fermions with distinct chiralities, $e V_{x}^{s}=\mu_{R}^{\Uparrow}-\mu_{L}^{\Uparrow}-\left(\mu_{R}^{\Downarrow}-\mu_{L}^{\Downarrow}\right)$, we obtain in the linear response limit $I_{y}=\frac{e^{2}}{h} V_{x}^{s}$ and, as a consequence, $\left|\sigma_{x y}^{S}\right|=\frac{e^{2}}{h}$. Note that a charge voltage between the edges vanishes, $e V_{x}^{c}=\mu_{R}^{\Uparrow}-\mu_{L}^{\Uparrow}+\left(\mu_{R}^{\Downarrow}-\mu_{L}^{\Downarrow}\right)=0$. The perfect quantization of the spin Hall conductivity holds as long as the Dirac-like edge states remain inside the energy gap. Let us assume for simplicity that the ribbon is wide enough $\left[\gg\left(p_{0,1,2}\right)^{-1}\right]$ to make negligibly small the interaction between opposite edges. As has been clearly shown just above, the edge state properties are essentially influenced by EP, which is present at the side boundaries of the ribbon-shaped sample. In particular, one can see in Fig. 7, that when the TI film is in the topological phase, $b \Delta_{0}>0$, and fermions propagating along the edges are subjected to the action of strong or weak EP, the edge state spectrum of Eq. (30) crosses the hybridization gap. In this case, the sample is expected to exhibit a pronounced conductance plateau of $\left|\sigma_{x y}^{S}\right|=\frac{e^{2}}{h}$ over a large range of the gate voltage [namely at $|\mu \lesssim 2| \Delta_{0} \mid \sqrt{\Lambda(1-\Lambda)}$ ]. At the same time, when EP has a moderate strength, the energy region, in which the edge state exists inside the gap, narrows and shifts to the $2 \mathrm{D}$ band continuum and can actually merge into the continuum at $\tilde{u} \approx 1$. Therefore, the quantized conductance plateau becomes less pronounced. In turn, as seen in Fig. 8, when the TI film is in the normal phase, $b \Delta_{0}<0$, an EP of the moderate strength, $\tilde{u} \approx 1$, provides the appearance of the Dirac-like edge states inside the gap. And these states could manifest themselves in the conductivity plateau, although this plateau is narrow. These features reflect a specific behavior of QSHE in the samples designed in the Hall bar geometry when $\mathrm{EP}$ at the edges is taken into account, as compared with the behavior considered solely in the terms of the Chern number. A possibility to observe the conductance plateau as a QSHE hallmark depends essentially on the conditions at the edges of the NI/TI/NI trilayer sample. Note that a regime with nonzero but nonquantized spin Hall conductivity could exist under less rigid restrictions. In particular, when the chemical potential lies beyond the hybridization gap, the spin Hall conductance is dominated by electron states of the 2 D continuum $E_{ \pm}(\kappa)$ of Eq. (25), which leads to perfect quantization break.

Our model of NI/TI/NI trilayer predicts evidence for the fact that the QSHE regime is highly sensitive to the conditions at both the interfacial and lateral borders of the TI film. We establish that in this system the QSHE realization is not connected unambiguously with the topological index of the TI film but depends on EP at the edges. The edge state properties (e.g., the spectrum and the space profile) can be substantially modified by EP, this leads to the appearance and disappearance of the spin-filtered current channels along the edges. One can choose the EP parameters in such a way that the edges do not host the helical channels with the Dirac-like spectrum inside the hybridization gap even in the topological insulating phase with $\left|C^{S}\right|=1$. On the other hand, in the trivial insulating phase with $C^{S}=0$, under EP of certain strength, there exist metallic edge states with the Dirac node inside the gap. Thus, in the present model of the NI/TI/NI trilayer, the QSHE manifestation is determined in a quite specific way by the geometry and physics of the boundaries of the TI film.

\section{DISCUSSION}

In the previous sections we argued that both the interfaces and the edges play an important role in spin transport properties of the NI/TI/NI trilayer. In accordance with the phase diagram in Fig. 6, the microscopic characteristics of the trilayer, $l$ and $U$, can vary significantly, while the topological index $C^{S}$ does not change as long as the product $b(l, U) \Delta_{0}(l, U)$ keeps the sign. However, this circumstance does not imply undoubtedly the existence of the conducting edge channels in the trilayer sample installed in a Hall bar geometry. Generally speaking, to understand deeper the relation of QSHE to boundary effects in realistic TI/NI heterostructures it is required to directly examine the sample by bulk-sensitive and scanning probe techniques and spin Hall response measurement simultaneously.

Our treatment of the QSHE problem is distinct from most of the ones discussed previously [14,26-28,30-32] since we incorporate the effect of the TI/NI interfaces and the edges of the TI film through the corresponding natural boundary conditions featured by the IP or EP [see Eqs. (5) and (29)], respectively. We demonstrate, by the example of the NI/TI/NI trilayer, that the interface and edge states and, as a consequence, the QSHE regime can be manipulated by changing the conditions at the TI film boundaries. This theoretical finding significantly enlarges possibilities to predict and analyze the manifestation of spin Hall effect for a broad class of heterostructures including semiconductors with strong spin orbit coupling [47-49]. Our results provide a rich playground to study boundary-originated changes in peculiar electron properties of various TI/NI heterostructures. So, under corresponding generalization, our approach can be applied to describe quantum anomalous Hall effect (QAHE) in the TI/NI heterostructure containing magnetically ordered constituents. One can explore a model of a NI/FMTI/NI trilayer with a 
ferromagnetic (FM) TI film adding an exchange term to the initial bulk Hamiltonian Eq. (2), as it had been done, for instance, in Refs. [50,51]. It is also possible to consider a model of a FMNI/TI/FMNI trilayer, in which the TI film is sandwiched with FM dielectrics.

Our study also concerns other aspect of the finite-size phenomena in semiconductor heterostructures. It was lately proposed that robust helical surface states can emerge in topologically trivial crystal systems at specific surfaces and under a proper combination of anisotropy and thickness [52-54]. Recently, Zhu et al. reported on the signature of such surface states in epitaxial $\mathrm{Bi}(111)$ thin films [55]. In the present study we mostly focus on a role of the effective boundary potentials in the helical states formation. Xu et al. have reported on the observation of quasi 2D states at the surface of $\mathrm{BiTl}\left(\mathrm{S}_{1-\delta} \mathrm{Se}_{\delta}\right)_{2}$ alloy, which resemble the helical states on the 3D TI surface, on the trivial side (with noninverted bulk spectrum) of the topological phase transition in the composition $\delta$ near the critical point $\delta \approx 0.4-0.5$ [56]. In Sec. VI we have found that the certain EP can induce the edge state in the bounded trilayer when the TI film is in the topologically trivial phase. Heretofore, it was analytically shown in Ref. [38] that, under certain boundary conditions, the Dirac-like surface states can appear in a truncated 3D insulator, which has non-inverted spectrum. These results provide further physical insight into the properties of time-reversal invariant insulators. Obviously, the analytic results are needed to be supported by numerical modeling ( $a b$ initio and/or tight-binding calculations) and transport measurements.

As follows from the above analysis, on the one hand, the closing and reopening of the hybridization gap $\Delta_{0}$ is accompanied by the band inversion of the $\varphi$ - and $\chi$-states (Fig. 1) and causes a jump of the spin Chern number $C^{S}$ (Fig. 6). On the other hand, the alternation of the sign of the dispersion parameter $b$, also leads to a change of $C^{S}$ (Fig. 6). It means that in our model the topological quantum phase transitions in the NI/TI/NI trilayer can befall both with and without closing and reopening of the gap. This fact has a formal foundation. According to Refs. [57,58], the Hamiltonian of the type $\mathcal{F}^{\Sigma}(\boldsymbol{\kappa})$, Eq. (12), belongs to a class of two-band continuous Hamiltonians possessing a quantum duality property. This means that the Hamiltonians $\mathcal{F}^{\Sigma}(\boldsymbol{\kappa})$ and $\widehat{\mathcal{F}}^{\Sigma}(\boldsymbol{\kappa})$ are dual each other provided that $\frac{\Delta_{0}}{b}$ is replaced by $\frac{b}{\Delta_{0}}$. In conformity with the duality property, $\mathcal{F}^{\Sigma}(\kappa)$ and $\widehat{\mathcal{F}}^{\Sigma}(\boldsymbol{\kappa})$ describe the same phase; whether this phase is topological or trivial it is determined by the sign of $b \Delta_{0}$.

Above in Sec. V we have estimated the distance between neighboring phase boundaries in the diagrams in Figs. 6 as $\frac{l_{0}}{4} \simeq 1.3 \mathrm{~nm}$. This characteristic scale is not much larger than the one quintuple layer (QL) thickness $(\approx 0.9 \mathrm{~nm})$ in the classic tetradymite TIs, which indicates that the QSHE regime is highly sensitive to variation of the TI film thickness. In practice, the fabricated TI/NI interfaces are not perfectly flat due to an unavoidable roughness. So, in the case of tetradymite semiconductors, the interface landscape is represented by terraces separated by steps running along different hexagonal axes. The step height is normally equal to one $\mathrm{QL}$, the value of the root-mean-square roughness can attain $2 \mathrm{~nm}$ [59]. The interface roughness directly corresponds to fluctuations of the
TI film thickness. Therefore, one can suggest that the TI film interior breaks down into distinct phases, with different $C^{S}$ equal to 0 or \pm 1 , separated from each other by the steps which can host $1 \mathrm{D}$ conducting channels. In terms of electrophysics the TI/NI interface can be viewed as a random network of the $1 \mathrm{D}$ channels possessing the conductance of $\pm \frac{e^{2}}{h}$. If the network forms a global conducting cluster, a percolation contribution to a total conductivity of a sample appears. Hereby, the presence of the interface roughness is an important point in the experimental search for high-performance spin transport materials and devices based on the TI/NI heterostructures.

In the present work, we have explored only the ideal model of the symmetric NI/TI/NI trilayer, which is rather appropriate to describe "an elementary cell" of superlattice heterostructures. In the case of real trilayers (or bilayers), the bottom surface of the TI film is usually attached to an NI substrate, while the top surface is either covered by an NI protective overlayer or exposed to a vacuum, which breaks the inversion symmetry along the growth direction, leading to the Rashba-like energy splitting for the gapped spectrum in the TI film. Furthermore, in the presence of the structural inversion asymmetry the Chern number loses its meaning since the 2D effective Hamiltonian Eq. (11) cannot be divided into two blocks. Nevertheless, the authors of Refs. [14,32,60] have proved that one can use the $Z_{2}$ topological classification to distinguish the topological phase from the trivial phase. Their calculations $[14,32,60]$ demonstrated that the system undergoes a topological phase transition to a normal insulator when the inversion asymmetry is large enough and/or the film is thin enough. In our approach, the broken structural inversion symmetry in the trilayer can be taken into account by setting different IPs at the opposite interfaces of the TI film.

\section{CONCLUSION}

In this paper, we have systematically investigated the effects of the interface and edge potentials as well as the TI film thickness on the electron properties of the NI/TI/NI trilayer. The energy spectrum and space distribution of the in-gap electron states are found to be very sensitive to these key attributes of the system. We have depicted the phase diagram for the NI/TI/NI trilayer unbound in the interface plane, which demonstrates the interface-driven transitions between topologically distinct phases. The EP effect on the behavior of the in-gap edge states at the edges of the trilayer finite in the interface plane is also highlighted. We have provided a physical explanation on how the spin Hall conductivity can be controlled in the TI/NI heterostructures. We have established that the realization of QSHE regime in the trilayer sample is intimately connected with interplay between the 2D interface-induced states and the 1D Dirac-like edge states. For the QSHE regime to take place it is essential that the Dirac point of the $1 \mathrm{D}$ edge states lies inside the hybridization gap of the $2 \mathrm{D}$ interface-induced states. Under relevant position of the chemical potential, carriers in the states propagating along the edges of the TI film provide spin conductivity in transport measurements. We have shown that the IP, in addition to the TI film thickness, drives the interface states in the film and, consequently, the condition for quantum phase 
transitions associated with the alternating-sign product $b \Delta_{0}$. In turn, the EP at the edges regulates the conducting edge states so they can occur inside the gap at both $b \Delta_{0}>0$ and $b \Delta_{0}<0$ depending on the EP strength. Therefore, the intrinsic spin conductivity in the Hall-bar sample is no longer related directly to the spin Chern number, which merely distinguishes the topological insulating phase $\left(b \Delta_{0}>0\right.$ and $\left.\left|C^{S}\right|=1\right)$ from the trivial insulating $\left(b \Delta_{0}<0\right.$ and $\left.C^{S}=0\right)$ phase in the ideal trilayer that is infinite in the interface plane. That is to say, in the NI/TI/NI trilayer, one can choose such EP (which does not break the time-reversal symmetry) that the nontrivial spin Chern number, $\left|C^{S}\right|=1$, does not manifest itself in QSHE owing to the absence of the in-gap conducting channels at edges of the TI film. On the contrary, under the trivial insulating regime, $C^{S}=0$, the $\mathrm{EP}$ of certain moderate strength can induce metallic helical channels that contribute to the QSHE transport. Nevertheless, it should be noted that, in the case of EP with the large or small strength, the edge states are guaranteed to reside in the hybridization gap under the stipulation $b \Delta_{0}>0$, and they are absent under $b \Delta_{0}<0$. Thus, we have reveal a essential role of boundary factors in the TI/NI heterostructures.

The realization of QSHE and its characteristics are tunable by changing the strength of both the interface potential and the edge potential, which makes the heterostructure design a versatile and powerful tool for spintronic applications of TIs. Our conclusions are relevant for a broad range of the heterostructures, including the NI/TI/NI trilayers and the [TI/NI] superlattices. Although we have limited ourselves to the study of QSHE, our approach can be generalized to describe quantum anomalous Hall effect in the TI/NI heterostructures, where TI or/and NI possesses magnetic ordering.

\section{ACKNOWLEDGMENTS}

We acknowledge partial support from the Basque Country Government, Departamento de Educación, Universidades e Investigación (Grant No. IT-756-13), the Spanish Ministerio de Ciencia e Innovación (Grant No. FIS2010-19609-C02-01), the Tomsk State University Academic D.I. Mendeleev Fund Program (Grant No. 8.1.05.2015), Saint Petersburg State University (Grant No. 15.61.202.2015), and Russian Foundation for Basic Research (Grant No. 16-02-00024). Numerical calculations were partly performed using computational resources provided by Resource Center "Computer Center of SPbU" (http://cc.spbu.ru), and the SKIF-Cyberia supercomputer at the National Research Tomsk State University.
[1] M. J. Brahlek, N. Koirala, J. Liu, T. I. Yusufaly, M. Salehi, M.-G. Han, Y. Zhu, D. Vanderbilt, and S. Oh, Tunable inverse topological heterostructure utilizing $\left(\mathrm{Bi}_{1-x} \mathrm{In}_{x}\right)_{2} \mathrm{Se}_{3}$ and multichannel weak-antilocalization effect, Phys. Rev. B 93, 125416 (2016).

[2] X.-G. Li, G.-F. Zhang, G.-F. Wu, H. Chen, C. Dimitrie, and Z.-Y. Zhang, Proximity effects in topological insulator heterostructures, Chin. Phys. B 22, 097306 (2013).

[3] Z. Chen, L. Zhao, K. Park, T. A. Garcia, M. C. Tamargo, and L. Krusin-Elbaum, Robust Topological Interfaces and Charge Transfer in Epitaxial $\mathrm{Bi}_{2} \mathrm{Se}_{3} / \mathrm{II}-\mathrm{VI}$ Semiconductor Superlattices, Nano Lett. 15, 6365 (2015).

[4] K. Nakayama, K. Eto, Y. Tanaka, T. Sato, S. Souma, T. Takahashi, K. Segawa, and Y. Ando, Manipulation of Topological States and the Bulk Band Gap Using Natural Heterostructures of a Topological Insulator, Phys. Rev. Lett. 109, 236804 (2012).

[5] T.-A. Nguyen, D. Backes, A. Singh, R. Mansell, C. Barnes, D. A. Ritchie, G. Mussler, M. Lanius, D. Grützmacher, and V. Narayan, Topological states and phase transitions in $\mathrm{Sb}_{2} \mathrm{Te}_{3}-$ GeTe multilayers, Sci. Rep. 6, 27716 (2016).

[6] T. Rauch, M. Flieger, J. Henk, and I. Mertig, Nontrivial interface states confined between two topological insulators, Phys. Rev. B 88, 245120 (2013).

[7] N. Koirala, M. Brahlek, M. Salehi, L. Wu, J. Dai, J. Waugh, T. Nummy, M.-G. Han, J. Moon, Y. Zhu, D. Dessau, W. $\mathrm{Wu}$, N. P. Armitage, and S. Oh, Record surface state mobility and quantum hall effect in topological insulator thin films via interface engineering, Nano Lett. 15, 8245 (2015).

[8] Y. Zhao, H. Liu, X. Guo, Y. Jiang, Y. Sun, H. Wang, Y. Wang, H.-D. Li, M.-H. Xie, X.-C. Xie, and J. Wang, Crossover from 3D to $2 \mathrm{D}$ quantum transport in $\mathrm{Bi}_{2} \mathrm{Se}_{3} / \mathrm{In}_{2} \mathrm{Se}_{3}$ superlattices, Nano Lett. 14, 5244 (2014).
[9] M. Z. Hasan and C. L. Kane, Colloquium: Topological insulators, Rev. Mod. Phys. 82, 3045 (2010).

[10] X.-L. Qi and S.-C. Zhang, Topological insulators and superconductors, Rev. Mod. Phys. 83, 1057 (2011).

[11] Y. Ando, Topological insulator materials, J. Phys. Soc. Jpn. 82, 102001 (2013).

[12] B. A. Bernevig, T. L. Hughes, and S.-C. Zhang, Quantum spin hall effect and topological phase transition in HgTe quantum wells, Science 314, 1757 (2006).

[13] B. Zhou, H.-Z. Lu, R.-L. Chu, S.-Q. Shen, and Q. Niu, Finite Size Effects on Helical Edge States in a Quantum Spin-Hall System, Phys. Rev. Lett. 101, 246807 (2008).

[14] H.-Z. Lu, W.-Y. Shan, W. Yao, Q. Niu, and S.-Q. Shen, Massive Dirac fermions and spin physics in an ultrathin film of topological insulator, Phys. Rev. B 81, 115407 (2010).

[15] C. Liu, T. L. Hughes, X.-L. Qi, K. Wang, and S.-C. Zhang, Quantum Spin Hall Effect in Inverted Type-II Semiconductors, Phys. Rev. Lett. 100, 236601 (2008).

[16] Y. Zhang, K. He, Y. Zhang, K. He, C.-Z. Chang, C.-L. Song, L.-L. Wang, X. Chen, J.-F. Jia, Z. Fang, X. Dai, W.-Y. Shan, S.-Q. Shen, Q. Niu, X.-L. Qi, S.-C. Zhang, X.-C. Ma, and Q.-K. Xue, Crossover of the three-dimensional topological insulator $\mathrm{Bi}_{2} \mathrm{Se}_{3}$ to the two-dimensional limit, Nat. Phys. 6, 584 (2010).

[17] Y. Sakamoto, T. Hirahara, H. Miyazaki, S. I. Kimura, and S. Hasegawa, Spectroscopic evidence of a topological quantum phase transition in ultrathin $\mathrm{Bi}_{2} \mathrm{Se}_{3}$ films, Phys. Rev. B 81, 165432 (2010).

[18] A. A. Taskin, S. Sasaki, K. Segawa, and Y. Ando, Manifestation of Topological Protection in Transport Properties of Epitaxial $\mathrm{Bi}_{2} \mathrm{Se}_{3}$ Thin Films, Phys. Rev. Lett. 109, 066803 (2012).

[19] L. Wu, M. Brahlek, R. V. Aguilar, A. V. Stier, C. M. Morris, Y. Lubashevsky, L. S. Bilbro, N. Bansal, S. Oh, and N. P. 
Armitage, A sudden collapse in the transport lifetime across the topological phase transition in $\left(\mathrm{Bi}_{1-x} \mathrm{In}_{x}\right)_{2} \mathrm{Se}_{3}$, Nat. Phys. 9, 410 (2013).

[20] D. Kim, P. Syers, N. P. Butch, J. Paglione, and M. S. Fuhrer, Coherent topological transport on the surface of $\mathrm{Bi}_{2} \mathrm{Se}_{3}$, Nat. Commun. 4, 2040 (2013).

[21] M. Neupane, A. Richardella, J. Sánchez-Barriga, S.-Y. Xu, N. Alidoust, I. Belopolski, C. Liu, G. Bian, D. Zhang, D. Marchenko, A. Varykhalov, O. Rader, M. Leandersson, T. Balasubramanian, T.-R. Chang, H.-T. Jeng, S. Basak, H. Lin, A. Bansil, N. Samarth, and M. Z. Hasan, Observation of quantum-tunneling modulated spin texture in ultrathin topological insulator $\mathrm{Bi}_{2} \mathrm{Se}_{3}$ films, Nat. Commun. 5, 3841 (2014).

[22] G. Landolt, S. Schreyeck, S. V. Eremeev, B. Slomski, S. Muff, J. Osterwalder, E. V. Chulkov, C. Gould, G. Karczewski, K. Brunner, H. Buhmann, L. W. Molenkamp, and J. H. Dil, Spin Texture of $\mathrm{Bi}_{2} \mathrm{Se}_{3}$ Thin Films in the Quantum Tunneling Limit, Phys. Rev. Lett. 112, 057601 (2014).

[23] Y. Jiang, Y. Wang, M. Chen, Z. Li, C. Song, K. He, L. Wang, X. Chen, X. Ma, and Q.-K. Xue, Landau Quantization and the Thickness Limit of Topological Insulator Thin Films of $\mathrm{Sb}_{2} \mathrm{Te}_{3}$, Phys. Rev. Lett. 108, 016401 (2012).

[24] J. Tominaga, R. E. Simpson, P. Fons, and A. V. Kolobov, Electrical-field induced giant magnetoresistivity in (nonmagnetic) phase change films, Appl. Phys. Lett. 99, 152105 (2011).

[25] I. Belopolski, S.-Y. Xu, N. Koirala, C. Liu, G. Bian, V. N. Strocov, G. Chang, M. Neupane, N. Alidoust, D. Sanchez, H. Zheng, M. Brahlek, V. Rogalev, T. Kim, N. C. Plumb, C. Chen, F. Bertran, P. Le Fèvre, A. Taleb-Ibrahimi, M.-C. Asensio, M. Shi, H. Lin, M. Hoesch, S. Oh, and M. Z. Hasan, A novel artificial condensed matter lattice and a new platform for one-dimensional topological phases, Sci. Adv. 3, e1501692 (2017).

[26] K. Ebihara, K. Yada, A. Yamakage, and Y. Tanaka, Finite-size effects of the surface states in a lattice model of topological insulator, Physica E (Amsterdam) 44, 885 (2012).

[27] K.-I. Imura, M. Okamoto, Y. Yoshimura, Y. Takane, and T. Ohtsuki, Finite-size energy gap in weak and strong topological insulators, Phys. Rev. B 86, 245436 (2012).

[28] M. Okamoto, Y. Takane, and K.-I. Imura, One-dimensional topological insulator: A model for studying finite-size effects in topological insulator thin films, Phys. Rev. B 89, 125425 (2014).

[29] T. Förster, P. Krüger, and M. Rohlfing, Two-dimensional topological phases and electronic spectrum of $\mathrm{Bi} 2 \mathrm{Se} 3$ thin films from GW calculations, Phys. Rev. B 92, 201404 (2015).

[30] J. Linder, T. Yokoyama, and A. Sudbo, Anomalous finite size effects on surface states in the topological insulator $\mathrm{Bi}_{2} \mathrm{Se}_{3}$, Phys. Rev. B 80, 205401 (2009).

[31] C. X. Liu, H. J. Zhang, B. H. Yan, X. L. Qi, T. Frauenheim, X. Dai, Z. Fang, and S. C. Zhang, Oscillatory crossover from twodimensional to three-dimensional topological insulators, Phys. Rev. B 81, 041307(R) (2010).

[32] W.-Y. Shan, H.-Z. Lu, and S.-Q. Shen, Effective continuous model for surface states and thin films of three-dimensional topological insulators, New J. Phys. 12, 043048 (2010).

[33] I. A. Nechaev, S. V. Eremeev, E. E. Krasovskii, P. M. Echenique, and E. V. Chulkov, Quantum spin Hall insulators in centrosymmetric thin films composed from topologically trivial BiTeI trilayers, Sci. Rep. 7, 43666 (2017).
[34] I. V. Silkin, Yu. M. Koroteev, S. V. Eremeev, G. Bihlmayer, and E. V. Chulkov, Three- and Two-Dimensional Topological Insulators in $\mathrm{Pb}_{2} \mathrm{Sb}_{2} \mathrm{Te}_{5}, \mathrm{~Pb}_{2} \mathrm{Bi}_{2} \mathrm{Te}_{5}$, and $\mathrm{Pb}_{2} \mathrm{Bi}_{2} \mathrm{Se}_{5}$ Layered Compounds, JETP Lett. 94, 217 (2011).

[35] A. Medhi and V. B. Shenoy, Continuum theory of edge states of topological insulators: variational principle and boundary conditions, J. Phys.: Condens. Matter 24, 355001 (2012).

[36] P. Michetti, P. H. Penteado, J. C. Egues, and P. Recher, Helical edge states in multiple topological mass domains, Semicond. Sci. Technol. 27, 124007 (2012).

[37] V. N. Men'shov, V. V. Tugushev, and E. V. Chulkov, Interface induced states at the boundary between a 3D topological insulator and a normal insulator, JETP Lett. 97, 258 (2013).

[38] V. N. Men'shov, V. V. Tugushev, T. V. Menshchikova, S. V. Eremeev, P. M. Echenique, and E. V. Chulkov, Modelling near-surface bound electron states in a 3D topological insulator: Analytical and numerical approaches, J. Phys.: Condens. Matter 26, 485003 (2014).

[39] V. N. Men'shov, V. V. Tugushev, S. V. Eremeev, P. M. Echenique, and E. V. Chulkov, Band bending driven evolution of the bound electron states at the interface between a three-dimensional topological insulator and a three-dimensional normal insulator, Phys. Rev. B 91, 075307 (2015).

[40] V. N. Men'shov, V. V. Tugushev, and E. V. Chulkov, Spin Hall Conductivity in Three-Dimensional Topological Insulator/Normal Insulator Heterostructures, JETP Lett. 102, 754 (2015).

[41] S.-Q. Shen, Topological Insulators (Springer, Berlin, 2012).

[42] H. J. Zhang, C. X. Liu, X. L. Qi, X. Dai, Z. Fang, and S. C. Zhang, Topological insulators in $\mathrm{Bi}_{2} \mathrm{Se}_{3}, \mathrm{Bi}_{2} \mathrm{Te}_{3}$ and $\mathrm{Sb}_{2} \mathrm{Te}_{3}$ with a single Dirac cone on the surface, Nat. Phys. 5, 438 (2009).

[43] V. V. Enaldiev, I. V. Zagorodnev, and V. A. Volkov, Boundary conditions and surface state spectra in topological insulators, JETP Lett. 101, 89 (2015).

[44] H. Weng, R. Yu, X. Hu, X. Dai, and Z. Fang, Quantum anomalous Hall effect and related topological electronic states, Adv. Phys. 64, 227 (2015).

[45] D. N. Sheng, Z. Y. Weng, L. Sheng, and F. D. M. Haldane, Quantum Spin-Hall Effect and Topologically Invariant Chern Numbers, Phys. Rev. Lett. 97, 036808 (2006).

[46] I. A. Nechaev and E. E. Krasovskii, Relativistic kp Hamiltonians for centrosymmetric topological insulators from $a b$ initio wave functions, Phys. Rev. B 94, 201410(R) (2016).

[47] H.-A. Engel, E. I. Rashba, and B. I. Halperin, Theory of spin Hall effects in semiconductors in Handbook of Magnetism and Advanced Magnetic Materials (Wiley, Chichester, 2007), Vol. 5, p. 2858.

[48] S. Murakami, N. Nagaosa, and S.-C. Zhang, Dissipationless Quantum Spin Current at Room Temperature, Science 301, 1348 (2003).

[49] S. Takahashi and S. Maekawa, Spin current, spin accumulation and spin Hall effect, Sci. Technol. Adv. Mater. 9, 014105 (2008).

[50] V. N. Men'shov, V. V. Tugushev, and E. V. Chulkov, Anomalous Hall and spin Hall conductivities in three-dimensional ferromagnetic topological insulator/normal insulator heterostructures, Europhys. Lett. 114, 37003 (2016).

[51] V. N. Men'shov, V. V. Tugushev, and E. V. Chulkov, Quantum anomalous Hall effect in magnetically modulated topological insulator/normal insulator heterostructures, JETP Lett. 104, 453 (2016). 
[52] H. Jiang, H. Liu, J. Feng, Q. Sun, and X. C. Xie, Transport Discovery of Emerging Robust Helical Surface States in $Z_{2}=0$ Systems, Phys. Rev. Lett. 112, 176601 (2014).

[53] Z. Wang, J. Song, H. Liu, H. Jiang, and X. C. Xie, Building topological devices through emerging robust helical surface states, New J. Phys. 17, 113040 (2015).

[54] H. Guo, Y. Lin, and S.-Q. Shen, Dimensional evolution between one- and two-dimensional topological phases, Phys. Rev. B 90, 085413 (2014).

[55] K. Zhu, L. Wu, X. Gong, S. Xiao, S. Li, X. Jin, M. Yao, D. Qian, M. Wu, J. Feng, Q. Niu, F. de Juan, and D.-H. Lee, The emergence of topologically protected surface states in epitaxial Bi(111) thin films, arXiv:1403.0066.

[56] S.-Y. Xu, M. Neupane, I. Belopolski, C. Liu, N. Alidoust, G. Bian, S. Jia, G. Landolt, B. Slomski, J. H. Dil, P. P. Shibayev, S. Basak, T.-R. Chang, H.-T. Jeng, R. J. Cava, H. Lin, A. Bansil, and
M. Z. Hasan, Unconventional transformation of spin Dirac phase across a topological quantum phase transition, Nat. Commun. 6, 6870 (2015).

[57] T. Chern, Quantum Dualities and Quantum Anomalous Hall Phases with Arbitrary Large Chern Numbers, arXiv:1603.08517.

[58] T. Chern, Models for $d$ Wave Topological Superconductors and Quantum Anomalous Hall Effect with Arbitrary Large Chern Numbers, arXiv:1601.04630.

[59] N. V. Tarakina, S. Schreyeck, M. Luysberg, S. Grauer, C. Schumacher, G. Karczewski, K. Brunner, C. Gould, H. Buhmann, R. E. Dunin-Borkowski, and L. W. Molenkamp, Suppressing twin formation in $\mathrm{Bi}_{2} \mathrm{Se}_{3}$ thin films, Adv. Mater. Interfaces 1, 1400134 (2014).

[60] H. Li, L. Sheng, D. N. Sheng, and D. Y. Xing, Chern number of thin films of the topological insulator $\mathrm{Bi}_{2} \mathrm{Se}_{3}$, Phys. Rev. B 82, 165104 (2010) 